\title{
SINGULARITIES OF THE BIEXTENSION METRIC FOR FAMILIES OF ABELIAN VARIETIES
}

\author{
JOSÉ IGNACIO BURGOS GIL ${ }^{1}$, DAVID HOLMES ${ }^{2}$ and ROBIN DE JONG ${ }^{2}$ \\ ${ }^{1}$ Instituto de Ciencias Matemáticas (CSIC-UAM-UCM-UCM3), Calle Nicolás Cabrera 15, \\ Campus UAM, Cantoblanco, 28049 Madrid, Spain; \\ email: burgos@icmat.es \\ ${ }^{2}$ Mathematical Institute, Leiden University, PO Box 9512, 2300 RA Leiden, The Netherlands; \\ email: holmesdst@math.leidenuniv.nl, rdejong@math.leidenuniv.nl
}

Received 31 August 2016; accepted 10 June 2018

\begin{abstract}
In this paper we study the singularities of the invariant metric of the Poincaré bundle over a family of abelian varieties and their duals over a base of arbitrary dimension. As an application of this study we prove the effectiveness of the height jump divisors for families of pointed abelian varieties. The effectiveness of the height jump divisor was conjectured by Hain in the more general case of variations of polarized Hodge structures of weight -1 .
\end{abstract}

2010 Mathematics Subject Classification: 14H10 (primary); 11G50, 14D07 (secondary)

\section{Introduction}

1.1. Families of curves. By way of motivation of the general results in this paper, consider the following situation. Let $X$ be a smooth complex algebraic variety of dimension $n$, and let $\pi: Y \rightarrow X$ be a family of smooth projective curves parametrized by $X$. Let $A, B$ be two relative degree zero divisors on $Y \rightarrow X$, with disjoint support. To these divisors we can associate a function $h: X \rightarrow \mathbb{R}$, given by the archimedean component of the Néron height pairing

$$
h(x)=\left\langle A_{x}, B_{x}\right\rangle_{\infty}
$$

where $x \in X$. Let $X \hookrightarrow \bar{X}$ be a smooth compactification of $X$ with $D=\bar{X} \backslash X$ a normal crossings divisor. We are interested in the behavior of the function

(c) The Author(s) 2018. This is an Open Access article, distributed under the terms of the Creative Commons Attribution licence (http://creativecommons.org/licenses/by/4.0/), which permits unrestricted re-use, distribution, and reproduction in any medium, provided the original work is properly cited. 
$h$ close to the boundary divisor $D$. As is customary to do, we assume that the monodromy operators on the homology of the fibers of $Y \rightarrow X$ about all irreducible components of $D$ are unipotent. Let $x_{0}$ be a point of $\bar{X}$, and $U \stackrel{\sim}{\rightarrow} \Delta^{n}$ a small enough coordinate neighborhood of $x_{0}$ such that $D \cap U$ is given by $q_{1} \cdots q_{k}=0$. Thanks to a result of Brosnan and Pearlstein [6] (see also $[16,18]$ for the case where $X$ has dimension 1 ), there exist a continuous function $h_{0}: U \backslash D^{\text {sing }} \rightarrow \mathbb{R}$ and rational numbers $f_{1}, \ldots, f_{k}$ such that on $U \backslash D$ the equality

$$
h\left(q_{1}, \ldots, q_{n}\right)=h_{0}\left(q_{1}, \ldots, q_{n}\right)-\sum_{i=1}^{k} f_{i} \log \left|q_{i}\right|
$$

holds. Since $h_{0}$ is continuous on $U \backslash D^{\text {sing }}$, this determines the behavior of $h$ close to the smooth points of $D$. The question remains what happens when we approach a point of $D^{\text {sing }}$. In other words, what kind of singularities may $h_{0}$ have on $D^{\text {sing }}$ ?

From the work by Pearlstein [20] we find a more precise statement. Let $x_{0} \in \bar{X}$ be as above. Then there exists a homogeneous weight-one function $f \in \mathbb{Q}\left(x_{1}\right.$, $\left.\ldots, x_{k}\right)$ such that the following holds. Consider a holomorphic test curve $\bar{\phi}: \bar{C} \rightarrow$ $\bar{X}$ that has image not contained in $D$, a point $0 \in \bar{C}$ such that $\bar{\phi}(0)=x_{0}$, and a local analytic coordinate $t$ for $\bar{C}$ close to 0 . Assume that $\bar{\phi}$ is given locally by

$$
t \mapsto\left(t^{m_{1}} u_{1}(t), \ldots, t^{m_{k}} u_{k}(t), q_{k+1}(t), \ldots, q_{n}(t)\right),
$$

where $m_{1}, \ldots, m_{k}$ are nonnegative integers, $u_{1}, \ldots, u_{k}$ are invertible functions and $q_{k+1}, \ldots, q_{n}$ are arbitrary holomorphic functions. Then the asymptotic estimate

$$
h(\bar{\phi}(t))=b^{\prime}(t)-f\left(m_{1}, \ldots, m_{k}\right) \log |t|
$$

holds in a neighborhood of $0 \in \bar{C}$. Here $b^{\prime}$ is a continuous function that extends continuously over 0 .

Naively one might expect that the function $f$ is linear and $f\left(m_{1}, \ldots, m_{k}\right)$ is just a linear combination of the numbers $f_{i}$ with coefficients given by the multiplicities $m_{i}$ of the curve $\bar{C}$. In general, however this turns out not to be the case. Examples of nonlinear $f$ can be found in [3] and [8]. In [1,3] and [16] one finds a combinatorial interpretation of the function $f$ in terms of potential theory on the dual graphs of stable curves.

As a special case of one of the main results of this paper we will have a stronger asymptotic estimate. Namely

$$
h\left(q_{1}, \ldots, q_{n}\right)=b\left(q_{1}, \ldots, q_{n}\right)+f\left(-\log \left|q_{1}\right|, \ldots,-\log \left|q_{k}\right|\right)
$$

on $U \backslash D$, where $b: U \backslash D \rightarrow \mathbb{R}$ is a bounded continuous function that extends in a continuous manner over $U \backslash D^{\text {sing }}$. The boundedness of $b$ can be seen as 
a uniformity property on the asymptotic estimates for different test curves. In general, as shown by Example 3.3 below, the function $b$ cannot be extended continuously to $D^{\text {sing }}$, thus the boundedness of $b$ is the strongest estimate that can be hoped for.

As a concrete example of the shape of the function $f$, consider the stable curve $Y_{0}$ obtained by glueing two projective lines at zero and infinity, and marking the point (1:1) in both components. Let $\bar{Y} \rightarrow \bar{X}$ be a versal deformation of $Y_{0}$. The locus in $\bar{X}$ where the morphism $\bar{Y} \rightarrow \bar{X}$ is not smooth is a normal crossings divisor, locally defined by $q_{1} q_{2}=0$, say. The examples in [3] and [8] show that the function $f\left(x_{1}, x_{2}\right)$ is given, up to linear forms in $x_{1}$ and $x_{2}$, by $x_{1} x_{2} /\left(x_{1}+x_{2}\right)$.

One may ask for further properties of $h$. For example, a result of Hayama and Pearlstein [15, Theorem 1.18] implies that $h$ is locally integrable. Another question is whether the same can be said about the forms $\partial h$ and $\partial \bar{\partial} h$ and their powers. As seen in [8] in a case where $\bar{X}$ is two-dimensional this may lead to interesting intersection numbers between infinite towers of divisors. We plan to address this question in full generality in a subsequent work. In this paper we will focus on the one-dimensional case because it is the only case needed to treat Conjecture 1.2 below. Thus assume that the dimension of $X$ is one. Let $h_{0}$ be the function appearing in equation (1.1). Then we prove that the 1 -form $\partial h_{0}$ is locally integrable on $U$ with zero residue. Also the 2 -form $\partial \bar{\partial} h_{0}$ is locally integrable on $U$.

\subsection{Admissible variations of mixed Hodge structures. The correct general} setting for approaching these issues is to consider a variation of polarized pure Hodge structures $\boldsymbol{H}$ of weight -1 over $X$, see for instance [13] and [14]. Let $\boldsymbol{H}^{\vee}$ be the dual variation. Let $J(\boldsymbol{H}) \rightarrow X$ and $J\left(\boldsymbol{H}^{\vee}\right) \rightarrow X$ be the corresponding families of intermediate Jacobians. Then on $J(\boldsymbol{H}) \underset{X}{\times} J\left(\boldsymbol{H}^{\vee}\right)$ one has a Poincaré (biextension) bundle $\mathcal{P}=\mathcal{P}(\boldsymbol{H})$ with its canonical (biextension) metric. The polarization induces an isogeny of complex tori $\lambda: J(\boldsymbol{H}) \rightarrow J\left(\boldsymbol{H}^{\vee}\right)$. Let $v$, $\mu: X \rightarrow J(\boldsymbol{H})$ be two sections (with good behavior near $D$, more precisely admissible normal functions). Then we define

$$
L=\mathcal{P}_{\nu, \mu} \stackrel{\text { def }}{=}(\nu, \lambda \mu)^{*} \mathcal{P},
$$

as a metrized analytic line bundle on $X$. We put $\mathcal{P}_{v}=\mathcal{P}_{v, v}$. This 'diagonal' case will be of special interest to us. One important example, discussed at length in [14] and [6], is given by the normal function in $J\left(\bigwedge^{3} H_{1}\left(Y_{x}\right)\right)=H_{3}\left(J\left(Y_{x}\right)\right)$ associated to the Ceresa cycle $\left[Y_{x}\right]-\left[-Y_{x}\right]$ in $J\left(Y_{x}\right)$, for a family of curves $Y \rightarrow X$.

A second example is provided by the sections determined by two relative degree zero divisors $A, B$ on a family of smooth projective curves, as above. Let $\boldsymbol{H}$ be the 
variation of Hodge structure given by the homology of the fibers of the family of curves $Y \rightarrow X$. Then $J(\boldsymbol{H})$ is the usual Jacobian fibration associated to $Y \rightarrow X$. It is principally polarized in a canonical way. The divisors $A, B$ give rise to sections $v, \mu$ of $J(\boldsymbol{H}) \rightarrow X$. The Deligne pairing associates to the line bundles $\mathcal{O}_{Y}(A)$ and $\mathcal{O}_{Y}(B)$ a line bundle $\langle A, B\rangle$ on $X$, in a functorial and bimultiplicative way, see [10]. The line bundle $\langle A, B\rangle$ comes with a canonical rational section $s_{A, B}$, as well as a canonical Hermitian metric $\|\cdot\|_{A, B}$. The metric on $\langle A, B\rangle$ is determined by the archimedean height pairing. More precisely, we have the identity

$$
h(x)=\left\langle A_{x}, B_{x}\right\rangle_{\infty}=-\log \left(\left\|s_{A, B}\right\|_{A, B}(x)\right)
$$

for all $x \in X$. There is a canonical isometry

$$
\langle A, B\rangle^{\otimes(-1)} \stackrel{\sim}{\rightarrow} \mathcal{P}_{\nu, \mu} .
$$

Thus the singularity near $x_{0}$ of the biextension metric of the local rational section $s_{A, B}$ precisely gives the singularity of the function $h$ near $x_{0}$ as discussed above.

Returning to the general set-up, the result of Brosnan and Pearlstein [6, Theorems 24 and 79] is that some power $L^{\otimes N}$ extends as a continuously metrized

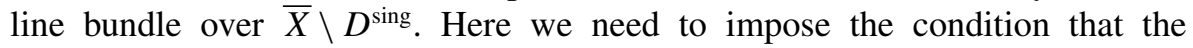
monodromy operators on the fibers of $\boldsymbol{H}$ about all irreducible components of $D$ are unipotent. Moreover, [6, Theorem 233 and Remark 234] provide a canonical extension of $L^{\otimes N}$ on $\bar{X} \backslash D^{\text {sing }}$ to an analytic line bundle over the whole of $\bar{X}$ (though the metric will in general not extend continuously over $D^{\text {sing }}$ ). Note that if the line bundle $L^{\otimes N}$ on $\bar{X} \backslash D^{\text {sing }}$ is algebraic, then it has a unique extension to an algebraic line bundle on $\bar{X}$. We denote the resulting line bundle on $\bar{X}$ by $\left[L^{\otimes N}\right.$, $\|-\|]_{\bar{X}}$. This extension is commonly known as the Lear extension of $L^{\otimes N}$, though the first general proof of its existence is due to Brosnan and Pearlstein in [6]. In order to remove the dependence on the choice of $N$ we will adopt the formalism of $\mathbb{Q}$-line bundles, and consider the Lear extension $[L,\|-\|]_{\bar{X}}=1 / N\left[L^{\otimes N},\|-\|\right]_{\bar{X}}$ as a $\mathbb{Q}$-line bundle on $\bar{X}$.

We are interested in the behavior of the biextension metric on $L$ when we approach a point $x_{0}$ in the singular locus $D^{\text {sing }}$. Let $s$ be a section of $L=\mathcal{P}_{v, \mu}$ on $U \cap X$ that corresponds to an admissible biextension variation of mixed Hodge structures. Pearlstein [20, Theorem 5.19] has proved that there exists a homogeneous weight-one function $f_{s} \in \mathbb{Q}\left(x_{1}, \ldots, x_{k}\right)$ such that for each holomorphic test curve $\bar{\phi}: \bar{C} \rightarrow \bar{X}$ as above the asymptotic estimate

$$
-\log \|s(\bar{\phi}(t))\|=b^{\prime}(t)-f_{s}\left(m_{1}, \ldots, m_{k}\right) \log |t|
$$

holds in a neighborhood $V$ of $0 \in \bar{C}$, with $b^{\prime}(t)$ continuous on $V$. 
Now assume that the polarized variation $\boldsymbol{H}$ is torsion-free and of type $(-1,0)$, $(0,-1)$ over $X$, so that the family $J(\boldsymbol{H}) \rightarrow X$ is a family of polarized abelian varieties over $X$. Under this assumption we are able to strengthen the result of Pearlstein's.

1.3. Statement of the main results. Recall that we work with a smooth complex algebraic variety $X$, provided with a partial compactification $\bar{X}$ with $D=\bar{X} \backslash X$ a normal crossings divisor, and a polarized pure variation of Hodge structures $\boldsymbol{H}$ of weight -1 over $X$.

Let $\left(q_{1}, \ldots, q_{n}\right): U \stackrel{\sim}{\rightarrow} \Delta^{n}$ be a coordinate chart on $\bar{X}$ such that $D \cap U=$ $\left\{q_{1} \cdots q_{k}=0\right\}$. Denote by $D_{i}$ the local component of $D$ with equation given by $q_{i}=0$. For any $0<\epsilon<1$ write

$$
U_{\epsilon}=\left\{\left(q_{1}, \ldots, q_{n}\right) \in U:\left|q_{i}\right|<\epsilon \text { for all } i=1, \ldots, n\right\} .
$$

Note that $U_{\epsilon} \cap X$ is identified via the coordinate chart with $\left(\Delta_{\epsilon}^{*}\right)^{k} \times \Delta_{\epsilon}^{n-k}$.

THEOREM 1.1. Assume that $\boldsymbol{H}$ is a variation of torsion-free polarized pure Hodge structures of type $(-1,0),(0,-1)$ on X. Assume that the monodromy operators on the fibers of $\boldsymbol{H}$ about the irreducible components of $D$ are unipotent. Let $v, \mu: X \rightarrow J(\boldsymbol{H})$ be two admissible normal functions of $J(\boldsymbol{H})$ over $X$. Then there exist an integer $d$, a homogeneous polynomial $Q \in \mathbb{Z}\left[x_{1}, \ldots, x_{k}\right]$ of degree $d$ with no zeros on $\mathbb{R}_{>0}^{k}$ and, for each section $s$ of $\mathcal{P}_{v, \mu}$ corresponding to an admissible biextension variation of mixed Hodge structures over $U \cap X$, a homogeneous polynomial $P_{s} \in \mathbb{Z}\left[x_{1}, \ldots, x_{k}\right]$ of degree $d+1$ such that the homogeneous weight-one rational function $f_{s}=P_{s} / Q$ satisfies the following properties.

(1) For all $\in \in \mathbb{R}_{>0}$ small enough, the function

$$
b\left(q_{1}, \ldots, q_{n}\right)=-\log \|s\|-f_{s}\left(-\log \left|q_{1}\right|, \ldots,-\log \left|q_{k}\right|\right)
$$

is bounded on $U_{\epsilon} \cap X$ and extends continuously over $U_{\epsilon} \backslash D^{\text {sing }}$.

(2) The function $f_{s}$ is uniquely determined by the previous property. Moreover, if $s^{\prime}$ is another section of $\mathcal{P}_{v, \mu}$ over $U \cap X$, such that

$$
\operatorname{div}\left(s^{\prime} / s\right)=\sum_{i=1}^{k} a_{i} D_{i},
$$

then the difference

$$
f_{s^{\prime}}-f_{s}=\sum_{i=1}^{k} a_{i}\left(-\log \left|q_{i}\right|\right)
$$

is linear in the functions $-\log \left|q_{i}\right|$. 
(3) The function $f_{s}: \mathbb{R}_{>0}^{k} \rightarrow \mathbb{R}$ extends to a continuous function $\bar{f}_{s}: \mathbb{R}_{\geqslant 0}^{k} \rightarrow \mathbb{R}$.

(4) In the case that $\mu=v$, the function $f_{s}$ is convex as a function on $\mathbb{R}_{>0}^{k}$ and the function $\bar{f}_{s}$ is convex as a function on $\mathbb{R}_{\geqslant 0}^{k}$.

We make a few remarks about Theorem 1.1. First of all, by [6, Theorem 81], if $U$ is small enough, admissible sections $s$ as in Theorem 1.1 exist.

Next, by [6, Corollary 177], if $U$ is small enough the set of admissible biextension variations on $U \cap X$ is a nonempty torsor over the group of meromorphic functions with poles only on $D$. Hence the admissibility of $s$ and condition (1.4) imply the admissibility of $s^{\prime}$.

Clearly, the function $f_{s}$ from Theorem 1.1 coincides with the $f_{s}$ from Pearlstein's asymptotic estimate (1.3). However, we do not assume [20, Theorem 5.19] in our proof, hence our arguments give an independent proof of (1.3) for the case of polarized, torsion-free variations of type $(-1,0),(0,-1)$.

If the family $J=J(\mathbf{H})$ of Jacobians is algebraic, that is, $J$ is an abelian scheme over $X$, then any two algebraic sections $\mu$ and $v$ of $J$ over $X$ are admissible, and for such $\mu, v$ the Lear extension of $\mathcal{P}_{v, \mu}$ over $\bar{X}$ is an algebraic $\mathbb{Q}$-line bundle.

Let the rank of $\boldsymbol{H}$ be $2 g$. Our proof of Theorem 1.1 in Section 4 will show that the function $f_{s}$ in the theorem has the shape

$$
f_{s}\left(x_{1}, \ldots, x_{k}\right)=\left(\sum_{i=1}^{k} x_{i} A_{i} c_{i}\right)^{t}\left(\sum_{i=1}^{k} x_{i} A_{i}\right)^{-1}\left(\sum_{i=1}^{k} x_{i} A_{i} c_{i}\right),
$$

where the $A_{i}(i=1, \ldots, k)$ are positive semidefinite $g \times g$ matrices such that $\sum_{i=1}^{k} A_{i}$ is positive definite, the $c_{i}$ are in $\mathbb{Q}^{g}$, and are determined by the monodromy of $\mu$ and $v$ about the branches of the divisor $D$. Thus the singularity of $-\log \|s\|$ has the shape

$$
\begin{aligned}
& f_{s}\left(-\log \left|q_{1}\right|, \ldots,-\log \left|q_{k}\right|\right) \\
& =\left(\sum_{i=1}^{k}-\log \left|q_{i}\right| A_{i} c_{i}\right)^{t}\left(\sum_{i=1}^{k}-\log \left|q_{i}\right| A_{i}\right)^{-1}\left(\sum_{i=1}^{k}-\log \left|q_{i}\right| A_{i} c_{i}\right) .
\end{aligned}
$$

Finally, Example 3.3 below will show that, in general, the locus of indeterminacy $D^{\text {sing }}$ of $b$ cannot be reduced to a smaller set.

We next turn to the issue of local integrability, in dimension one. Hain has made the following conjecture (see [14, Conjecture 6.4]). Assume we work with an arbitrary polarized variation of Hodge structures $(\boldsymbol{H}, \lambda)$ of weight -1 , whose underlying local system of abelian groups is torsion-free, and let $\mathcal{P}$ be its Poincaré bundle. Let $v$ be an admissible normal function of the family of intermediate Jacobians $J(\boldsymbol{H})$ over $X$. 
Conjecture 1.2 (Hain). Write $\hat{\mathcal{P}}=(\mathrm{id}, \lambda)^{*} \mathcal{P}$ and let $\omega=c_{1}(\hat{\mathcal{P}})$ be the first Chern form of the pullback of the Poincare bundle with its canonical metric. Assume that $X$ is a curve. Let $L=\mathcal{P}_{v}=v^{*} \hat{\mathcal{P}}$ with induced metric $\|-\|$ and let $N \in \mathbb{Z}_{>0}$ be such that $L^{\otimes N}$ extends as a continuous metrized line bundle over $\bar{X}$. Let $c_{1}\left(\left[L^{\otimes N},\|-\|\right]_{\bar{X}}\right)$ be the first Chern class of the extended line bundle [ $L^{\otimes N}$, $\|-\|]_{\bar{X}}$. Then the 2 -form $v^{*} \omega$ is integrable on $\bar{X}$, and the equality

$$
\int_{X} v^{*} \omega=\frac{1}{N} \int_{\bar{X}} c_{1}\left(\left[L^{\otimes N},\|-\|\right]_{\bar{X}}\right)
$$

holds.

Note that $v^{*} \omega=c_{1}\left(\mathcal{P}_{v}\right)$, and that the integral on the right hand side equals $1 / N \operatorname{deg}_{\bar{X}}\left[L^{\otimes N},\|-\|\right]_{\bar{X}}$. We prove the following result, which implies Hain's conjecture in the case of a variation of torsion-free polarized Hodge structure of type $(-1,0),(0,-1)$.

THEOREM 1.3. Assume that the polarized variation $\boldsymbol{H}$ over $X$ is torsion-free and pure of type $(-1,0),(0,-1)$, and that the monodromy operators on the fibers of $\boldsymbol{H}$ about all irreducible components of $D$ are unipotent. Let $s$ be a section of $\mathcal{P}_{\nu, \mu}$ corresponding to an admissible biextension variation of mixed Hodge structures over $U \cap X$ and assume that $\operatorname{dim} X=1$. Write

$$
-\log \|s\|=b(z)-r \log |t|
$$

on $U \cap X$ with $r \in \mathbb{Q}$ and with b bounded continuous on $U$, as can be done by the existence of the Lear extension of $\mathcal{P}_{v, \mu}$ over $\bar{X}$. Then the 1-form $\partial b$ is locally integrable on $U$ with zero residue. Moreover the 2 -form $\partial \bar{\partial} b$ is locally integrable on $U$.

As also $\partial \bar{\partial} \log |t|$ is locally integrable, we find that $\partial \bar{\partial} \log \|s\|$ is locally integrable. Since moreover the 1 -form $\partial b$ has no residue on $U$, so that $d[\bar{\partial} b]=[\partial \bar{\partial} b]$, upon globalizing using bump functions and applying Stokes' theorem we find

$$
\int_{X} c_{1}\left(\mathcal{P}_{\nu, \mu}\right)=\frac{1}{N} \int_{\bar{X}} c_{1}\left(\left[\mathcal{P}_{\nu, \mu}^{\otimes N},\|-\|\right]_{\bar{X}}\right)=\operatorname{deg}\left[\mathcal{P}_{\nu, \mu},\|-\|\right]_{\bar{X}} .
$$

In the diagonal case, we mention that by [14, Theorem 13.1] or [21, Theorem 8.2] the metric on $\mathcal{P}_{v}$ is nonnegative. Thus Theorem 1.3 implies that actually the inequality

$$
\operatorname{deg}\left[\mathcal{P}_{\nu},\|-\|\right]_{\bar{X}} \geqslant 0
$$


holds. We mention that in a letter to Griffiths, Pearlstein sketches a proof of Conjecture 1.2, and hence of the inequality (1.6), without the assumption that the type be $(-1,0),(0,-1)$.

We return again to the setting where the parameter space $X$ is of any dimension. However, we specialize to the 'diagonal' case where $\mu=v$. Consider, as before a test curve $\bar{\phi}: \bar{C} \rightarrow \bar{X}$ that has image not contained in $D$, and a point $0 \in \bar{C}$ such that $\bar{\phi}(0)=x_{0}$. Let $\phi$ denote the restriction of $\bar{\phi}$ to $\bar{C} \backslash \bar{\phi}^{-1} D$. The $\mathbb{Q}$-line bundle

$$
\left[\phi^{*}\left(\mathcal{P}_{\nu},\|-\|\right)\right]_{\bar{C}}^{\otimes-1} \otimes \bar{\phi}^{*}\left[\mathcal{P}_{\nu},\|-\|\right]_{\bar{X}}
$$

has a canonical nonzero rational section, as it is canonically trivial over $\bar{C} \backslash \bar{\phi}^{-1} D$. We call its divisor the height jump divisor $J=J_{\phi, v}$ on $\bar{C}$. R. Hain has made the following conjecture (see [14, end of Section 14]).

Conjecture 1.4. For all holomorphic test curves $\bar{\phi}: \bar{C} \rightarrow \bar{X}$ with image not contained in $D$, the height jump divisor $J=J_{\phi, v}$ on $\bar{C}$ is effective.

Choose coordinates in a neighborhood $U$ of $x_{0}$ as before so that $x_{0}$ has coordinates $(0, \ldots, 0)$ and let $f_{s} \in \mathbb{Q}\left(x_{1}, \ldots, x_{k}\right)$ be as in Pearlstein's asymptotic estimate (1.3), based on the choice of some admissible section $s$ of $\mathcal{P}_{v}$ on $U \cap X$. It can be shown that the function $f_{s}: \mathbb{R}_{>0}^{k} \rightarrow \mathbb{R}$ extends to a continuous function $\bar{f}_{s}: \mathbb{R}_{\geqslant 0}^{k} \rightarrow \mathbb{R}$. Locally around 0 the map $\bar{\phi}$ can be written as

$$
\bar{\phi}(t)=\left(t^{m_{1}} u_{1}(t), \ldots, t^{m_{k}} u_{k}(t), q_{k+1}(t), \ldots, q_{n}(t)\right),
$$

where, for $i \in[1, k], m_{i}>0$ and $u_{i}(0) \neq 0$. Write $\bar{f}_{s, i} \stackrel{\text { def }}{=} \bar{f}_{s}(0, \ldots, 0,1,0, \ldots, 0)$ (the 1 placed in the $i$ th spot), then

$$
\operatorname{ord}_{0} J=-\bar{f}_{s}\left(m_{1}, \ldots, m_{k}\right)+\sum_{i=1}^{k} m_{i} \bar{f}_{s, i} .
$$

Note that indeed $\operatorname{ord}_{0} J$ is independent of the choice of $s$. The rational number $\operatorname{ord}_{0} J$ is called the 'height jump' associated to the test curve $\bar{\phi}$, the admissible normal function $v$ and the point $0 \in \bar{C}$.

The terminology is due to Hain [14], who also observed a first instance where the height jump is nonzero. We refer the reader to the monograph [6] by Brosnan and Pearlstein, where an extensive study of the height jump in complete generality is given. Note that the height jumps precisely when $f_{s}$ is not linear. We mention that Conjecture 1.4 about the height jump was stated in [14] only for the normal function on $\mathcal{M}_{g}$ associated to the Ceresa cycle, but it seems reasonable to make this broader conjecture. 
In this paper we prove Conjecture 1.4 in the case of admissible normal functions of families of polarized abelian varieties.

THEOREM 1.5. Assume that the polarized variation $\boldsymbol{H}$ over the smooth complex variety $X$ is torsion-free and pure of type $(-1,0),(0,-1)$, and that the monodromy operators on the fibers of $\boldsymbol{H}$ about all irreducible components of $D$ are unipotent. Let $v$ be an admissible normal function of the family of intermediate Jacobians $J(\boldsymbol{H})$ over $X$. Then for all holomorphic test curves $\bar{\phi}: \bar{C} \rightarrow \bar{X}$ with image not contained in $D$, the associated height jump divisor $J=J_{\phi, v}$ on $\bar{C}$ is effective.

Combining with inequality (1.6) we obtain

COROllary 1.6. Assume that $\bar{C}$ is smooth and projective. Then under the assumptions of Theorem 1.5 , the $\mathbb{Q}$-line bundle $\bar{\phi}^{*}\left[\mathcal{P}_{\nu},\|-\|\right]_{\bar{X}}$ has nonnegative degree on $\bar{C}$.

The key to our proof of Theorem 1.5 is the convexity of the homogeneous function $\bar{f}_{s}$, as asserted in Theorem 1.1(4). We have the following explicit expression for $\operatorname{ord}_{0} J$. In equation (1.5) we already gave an expression for $f_{s}$ and hence $\bar{f}_{s}$ in terms of matrices $A_{i}$ and vectors $c_{i}$ for $i=1, \ldots, k$. We will see in subsection 3.4 that $\bar{f}_{s, i}=c_{i}^{t} A_{i} c_{i}$ for $i=1, \ldots, k$. Following the general expression (1.7) this gives

$$
\operatorname{ord}_{0} J=-\left(\sum_{i=1}^{k} m_{i} A_{i} c_{i}\right)^{t}\left(\sum_{i=1}^{k} m_{i} A_{i}\right)^{-1}\left(\sum_{i=1}^{k} m_{i} A_{i} c_{i}\right)+\sum_{i=1}^{k} m_{i} c_{i}^{t} A_{i} c_{i}
$$

for the height jump in our setting.

Turning again to the case of the Ceresa cycle, note that since the intermediate Jacobian of the primitive part of $H_{3}\left(J\left(Y_{x}\right)\right)$ is a compact complex torus but not an abelian variety, we cannot apply directly our results for families of abelian varieties to this case.

In the special case of families of Jacobians of curves Conjecture 1.4 has been proved in [3]. The proof in this special case makes heavy use of the combinatorics of dual graphs of nodal curves, and so cannot readily be extended to families of abelian varieties, nor does it seem practical to reduce the general case to that of Jacobians of curves.

REMARK 1.7. After the initial submission of the present paper to arXiv, two proofs of Conjecture 1.4 have appeared, see [6] and [7]. 
1.4. Overview of the paper. We review the content of the different sections of this paper. In the preliminary Section 2 we start by recalling the notions of $\mathbb{Q}$-line bundle and of Lear extension, and the Poincaré bundle on the product of a complex torus and its dual, together with its associated metric. We also recall the explicit description of the Poincaré bundle and its metric on a family of polarized abelian varieties. Also we study the period map associated to a family of pointed polarized abelian varieties. Moreover we give a local expansion for the metric on the pullback of the Poincare bundle under this period map. The functions that appear as the logarithm of the norm of a section of the pullback of the Poincare bundle will be called normlike functions.

In Section 3 we study normlike functions and give several estimates on their growth and that of their derivatives. Finally in Section 4 we prove the main results on local integrability and positivity of the height jump.

We fix some notation that we will use throughout. Let $r$ be a positive integer. For any commutative ring $R$ we will denote by $\operatorname{Col}_{r}(R)$ (respectively $\operatorname{Row}_{r}(R)$, $M_{r}(R)$ and $\left.S_{r}(R)\right)$ the set of column vectors of size $r$ with entries in $R$ (respectively row vectors, matrices and symmetric matrices of size $r$-by- $r$ ).

We denote by $S_{r}^{++}(\mathbb{R}) \subset S_{r}(\mathbb{R})$ (respectively $S_{r}^{+}(\mathbb{R}) \subset S_{r}(\mathbb{R})$ ) the cone of positive definite (respectively positive semidefinite) symmetric real matrices. We denote by $\mathbb{H}_{r}$ Siegel's upper half space of rank $r$, and by $\mathbb{P}^{r}$ its compact dual.

By a variety we mean an integral separated scheme of finite type over $\mathbb{C}$.

\section{Preliminary results}

2.1. Lear extensions. We start by recalling the formalism of $\mathbb{Q}$-line bundles. Details can be found in [3, Definition 2.10].

DEFINITION 2.1. Let $X$ be a complex variety. An (algebraic respectively analytic) $\mathbb{Q}$-line bundle over $X$ is a pair $(L, r)$ where $L$ is an (algebraic respectively analytic) line bundle on $X$ and $r>0$ is a positive integer (informally, we think of it as $\left.L^{\otimes 1 / r}\right)$. A metrized $\mathbb{Q}$-line bundle is a triple $(L,\|-\|, r)$, where $(L, r)$ is a $\mathbb{Q}$-line bundle and $\|-\|$ is a continuous metric on $L$. An isomorphism of $\mathbb{Q}$-line bundles $\left(L_{1}, r_{1}\right) \rightarrow\left(L_{2}, r_{2}\right)$ is an equivalence class of pairs $(a$, $f$ ) where $a$ is a positive integer and $f: L_{1}^{\otimes a r_{2}} \rightarrow L_{2}^{\otimes a r_{1}}$ is an isomorphism, where the equivalence relation is generated by setting $(a, f) \sim\left(a n, f^{\otimes n}\right)$. An isomorphism of metrized line bundles is an isometry if one (equivalently all) of the corresponding morphisms of line bundles is an isometry. Every line bundle $L$ gives rise to a $\mathbb{Q}$-line bundle $(L, 1)$. Note that, if $L$ is a line bundle and $r>1$ is an integer, then there is a canonical isomorphism $\left(L^{\otimes r}, r\right) \simeq(L, 1)$. Moreover, if $L$ is a torsion line bundle so that $L^{\otimes r} \simeq \mathcal{O}_{X}$, then there is an isomorphism of 
$\mathbb{Q}$-line bundles $(L, 1) \rightarrow\left(\mathcal{O}_{X}, r\right)$. If we do not need to specify the multiplicity $r$, a $\mathbb{Q}$-line bundle will be denoted by a single letter. We note that the group of isomorphism classes of $\mathbb{Q}$-line bundles on $X$ is equal to $\operatorname{Pic}(X) \otimes_{\mathbb{Z}} \mathbb{Q}$.

We denote

$$
\operatorname{Rat}_{\mathbb{Q}}(X)=(\mathcal{O}(X) \backslash\{0\}, \times) \otimes \mathbb{Q} .
$$

If $(L, r)$ is a $\mathbb{Q}$-line bundle, a $\mathbb{Q}$-rational section of $(L, r)$ (or rational section for short) is an equivalence class of symbols $s^{1 / r d}$, where $s$ is a nonzero rational section of $L^{\otimes d}$. Two symbols $s_{1}^{1 / r d_{1}}$ and $s_{2}^{1 / r d_{2}}$ are equivalent if

$$
\left(s_{1}\right)^{\otimes d_{2}}=\left(s_{2}\right)^{\otimes d_{1}}
$$

as a section of $L^{\otimes d_{1} d_{2}}$. The space of rational sections of $(L, r)$ is a torsor over $\operatorname{Rat}_{\mathbb{Q}}(X)$. Moreover, if $s$ and $s^{\prime}$ are rational sections of $(L, r)$ and $\left(L^{\prime}, r^{\prime}\right)$ then $s \otimes s^{\prime}$ is a rational section of $\left(L^{\otimes r^{\prime}} \otimes\left(L^{\prime}\right)^{\otimes r}, r r^{\prime}\right)$, but there is no additive structure of rational sections.

The divisor of the section $s^{1 / r d}$ is

$$
\operatorname{div}\left(s^{1 / r d}\right)=\frac{1}{r d} \operatorname{div}(s)
$$

Definition 2.2 (Lear extension). Let $X \subseteq \bar{X}$ be an open immersion of smooth complex varieties, such that the boundary divisor $D \stackrel{\text { def }}{=} \bar{X} \backslash X$ has normal crossings, and $L$ a line bundle on $X$ with continuous metric $\|-\|$. A Lear extension of $L$ is a $\mathbb{Q}$-line bundle $(\mathcal{L}, r)$ on $\bar{X}$ together with an isomorphism $\alpha$ : $(L$, $1)\left.\rightarrow(\mathcal{L}, r)\right|_{X}$ and a continuous metric on $\left.\mathcal{L}\right|_{\bar{X} \backslash D^{\text {sing }}}$ such that the isomorphism $\alpha$ is an isometry. Since $D^{\text {sing }}$ has codimension at least 2 in $\bar{X}$, if a Lear extension exists then it is unique up to a unique isomorphism. If a Lear extension of $L$ exists we denote it by $[L,\|-\|]_{\bar{X}}$. Note that the isomorphism class of the Lear extension of $L$ depends not only on $L$ but also on the metric on $L$.

If $s$ is a rational section of $L$, writing $s=\left(s^{\otimes r}\right)^{1 / r}$, it can also be seen as a rational section of $[L,\|-\|]_{\bar{X}}$. We will denote by $\operatorname{div}_{X}(s)$ the divisor of $s$ as a rational section of $L$ and by $\operatorname{div}_{\bar{X}}(s)$ the divisor of $s$ as a rational section of $[L,\|-\|]_{\bar{X}}$.

2.2. Poincaré bundle and its metric. In this section we recall the definition of the Poincaré bundle and its biextension metric. Moreover we make the biextension metric explicit in the case of families of polarized abelian varieties.

In the literature one can find small discrepancies in the description of the Poincaré bundle, see Remark 2.5. These discrepancies can be traced back to two different choices of the identification of a complex torus with its bidual. Moreover, 
there are also different conventions regarding the sign of the polarization of the abelian variety. Since one of our main results is a positivity result it is worthwhile to fix all the signs to avoid these ambiguities.

Complex tori and their duals. Let $g \geqslant 0$ be a nonnegative integer, $V$ a $g$ dimensional complex vector space and $\Lambda \subset V$ a rank-2 $g$ lattice. The quotient $T=V / \Lambda$ is a compact complex torus. It is a Kähler complex manifold, but in general it is not an algebraic variety.

We recall the construction of the dual torus of $T$. We denote by $V^{*}=\operatorname{Hom}_{\mathbb{C}}(V$, $\mathbb{C})$ the space of antilinear forms $w: V \rightarrow \mathbb{C}$. This is not the dual $V^{\vee}$ of $V$. In fact, let $\bar{V}$ denote the abelian group $V$ with the complex structure ${ }^{-}$given by

$$
\alpha^{-} v=\bar{\alpha} \cdot v
$$

Then $V^{*}=\bar{V}^{\vee}$.

The bilinear form

$$
\langle\cdot, \cdot\rangle: V^{*} \times V \rightarrow \mathbb{R},\langle w, z\rangle \stackrel{\text { def }}{=} \operatorname{Im}(w(z))
$$

is nondegenerate. Thus

$$
\Lambda^{\vee} \stackrel{\text { def }}{=}\left\{\lambda \in V^{*} \mid\langle\lambda, \Lambda\rangle \subset \mathbb{Z}\right\}
$$

is a lattice of $V^{*}$. The lattice $\Lambda^{\vee}$ is canonically isomorphic to the dual of the lattice $\Lambda$. The quotient $T^{\vee}=V^{*} / \Lambda^{\vee}$ is again a compact complex torus, called the dual torus of $T$.

We can identify $V$ with $\operatorname{Hom}_{\overline{\mathbb{C}}}\left(V^{*}, \mathbb{C}\right)$ by the rule

$$
z(w)=\overline{w(z)}
$$

so that the bilinear pairing

$$
\left(V^{*} \oplus V\right) \otimes\left(V^{*} \oplus V\right) \rightarrow \mathbb{R}, \quad(w, z) \otimes\left(w^{\prime}, z^{\prime}\right) \mapsto \operatorname{Im}\left(w\left(z^{\prime}\right)\right)+\operatorname{Im}\left(z\left(w^{\prime}\right)\right)
$$

is antisymmetric. With this identification the double dual $\left(T^{\vee}\right)^{\vee}$ gets identified with $T$.

The points of $T^{\vee}$ define homologically trivial line bundles on $T$ giving an isomorphism of $T^{\vee}$ with $\mathrm{Pic}^{0}(T)$. We recall this construction. Let $\mathbb{C}_{1}$ denote the subgroup of $\mathbb{C}^{\times}$of elements of norm one. Let $w \in V^{*}$. Denote by [ $\left.w\right]$ its class in $T^{\vee}$ and by $\chi_{[w]} \in \operatorname{Hom}\left(\Lambda, \mathbb{C}_{1}\right)$ the character

$$
\chi_{[w]}(\mu)=\exp (2 \pi i\langle w, \mu\rangle)
$$


The line bundle associated to $[w]$ is the line bundle $L_{[w]}$ with automorphy factor $\chi_{[w]}$. In other words, consider the action of $\Lambda$ on $V \times \mathbb{C}$ given by

$$
\mu(z, t)=(z+\mu, t \exp (2 \pi i\langle w, \mu\rangle)) .
$$

Write $L_{[w]}=(V \times \mathbb{C}) / \Lambda$. The projection $V \times \mathbb{C} \rightarrow V$ induces a map $L_{[w]} \rightarrow T$. It is easy to check that $L_{[w]}$ is a holomorphic line bundle on $T$ that only depends on the class $[w]$. Note that the identification between $T^{\vee}$ and $\operatorname{Pic}^{0}(T)$ is not completely canonical because it depends on a choice of sign. We could equally well have used the character $\chi_{[w]}^{-1}$.

The Poincaré bundle. Note that, although the cocycle equation (2.2) is not holomorphic in $w$, the line bundle $L_{[w]}$ varies holomorphically with $w$, defining a holomorphic line bundle on $T \times T^{\vee}$ called the Poincaré bundle. See [4, Section 2.5] for details.

Definition 2.3. A Poincaré (line) bundle $\mathcal{P}$ is a holomorphic line bundle on $T \times T^{\vee}$ that satisfies

(1) the restriction $\left.\mathcal{P}\right|_{T \times\{[w]\}}$ is isomorphic to $L_{[w]}$;

(2) the restriction $\left.\mathcal{P}\right|_{\{0\} \times T^{\vee}}$ is trivial.

A rigidified Poincaré bundle is a Poincaré bundle together with an isomorphism $\left.\mathcal{P}\right|_{\{0\} \times T^{\vee}} \stackrel{\sim}{\rightarrow} \mathcal{O}_{\{0\} \times T^{\vee}}$.

To prove the existence of a Poincaré bundle, consider the map

$$
a_{\mathcal{P}}:\left(\Lambda \times \Lambda^{\vee}\right) \times\left(V \times V^{*}\right) \rightarrow \mathbb{C}^{\times}
$$

given by

$$
a_{\mathcal{P}}((\mu, \lambda),(z, w))=\exp (\pi((w+\lambda)(\mu)+\overline{\lambda(z)})) .
$$

This map is holomorphic in $z$ and $w$. Moreover, since for $(\mu, \lambda) \in \Lambda \times \Lambda^{\vee}$,

$$
\langle\lambda, \mu\rangle=\frac{1}{2 i}(\lambda(\mu)-\overline{\lambda(\mu)}) \in \mathbb{Z},
$$

the map $a_{\mathcal{P}}$ is a cocycle for the additive action of $\Lambda \times \Lambda^{\vee}$ on $V \times V^{*}$. Hence, it is an automorphy factor that defines a holomorphic line bundle $\mathcal{P}$ on $T \times T^{\vee}=$ $V \times V^{*} / \Lambda \times \Lambda^{\vee}$.

For a fixed $w \in V^{*}$,

$$
a_{\mathcal{P}}((\mu, 0),(z, w))=\exp (\pi w(\mu)) .
$$


This last cocycle is equivalent to the cocycle equation (2.2). Indeed,

$$
\exp (\pi w(\mu)) \exp (\pi \overline{w(z+\mu)})^{-1} \exp (\pi \overline{w(z)})=\exp (2 \pi i\langle w, \mu\rangle),
$$

and the function $z \mapsto \exp (\pi \overline{w(z)})$ is holomorphic in $z$. Thus the restriction $\left.\mathcal{P}\right|_{T \times\{[w]\}}$ is isomorphic to $L_{[w]}$. Moreover

$$
a_{\mathcal{P}}((0, \lambda),(0, w))=1,
$$

which implies that the restriction $\left.\mathcal{P}\right|_{\{0\} \times T^{\vee}}$ is trivial. The uniqueness of the Poincaré bundle follows from the seesaw principle (see [4, Appendix A]).

We conclude

Proposition 2.4. A Poincaré bundle exists and is unique up to isomorphism. A rigidified Poincaré bundle exists and is unique up to a unique isomorphism.

REMARK 2.5. Using the above identification of $T$ with the dual torus of $T^{\vee}$ we have that, for a fixed $z \in V$, the restriction $\left.\mathcal{P}\right|_{\{[z]\} \times T^{\vee}}$ agrees with $L_{[z]}$. In fact

$$
a_{\mathcal{P}}((0, \lambda),(z, w))=\exp (\pi \overline{\lambda(z)}),
$$

and, arguing as in the proof of Proposition 2.4, this cocycle is equivalent to the cocycle

$$
\exp (2 \pi i \operatorname{Im}(\overline{\lambda(z)}))=\exp (2 \pi i\langle z, \lambda\rangle) .
$$

Note that the definition of the Poincaré bundle in [13, Section 3.2] states that $\left.\mathcal{P}\right|_{\{[z]\} \times T^{\vee}}=L_{[-z]}$. The discrepancy between [13] and the current paper is due to a different choice of identification between $T$ and $\left(T^{\vee}\right)^{\vee}$.

REMARK 2.6. As we will see later, in equation (2.12), the cocycle (2.3) is not optimal because it does not vary holomorphically in holomorphic families of tori.

Group theoretical interpretation of the Poincaré bundle. We next give a group theoretic description of the Poincare bundle. We start with the additive real Lie group $W$ given by

$$
W=V \times V^{*} .
$$

Denote by $\widetilde{W}$ the semidirect product $\widetilde{W}=W \ltimes \mathbb{C}^{\times}$, where the product in $\widetilde{W}$ is given by

$$
((z, w), t) \cdot\left(\left(z^{\prime}, w^{\prime}\right), t^{\prime}\right)=\left(\left(z+z^{\prime}, w+w^{\prime}\right), t t^{\prime} \exp \left(2 \pi i\left\langle w, z^{\prime}\right\rangle\right)\right) .
$$

Clearly the group

$$
W_{\mathbb{Z}}=\Lambda \times \Lambda^{\vee}
$$

is a subgroup of $\widetilde{W}$. 
Consider the space

$$
P \stackrel{\text { def }}{=} V \times V^{*} \times \mathbb{C}^{\times}
$$

and the action of $\widetilde{W}$ on $P$ by biholomorphisms given by

$$
((\mu, \lambda), t) \cdot((z, w), s)=(z+\mu, w+\lambda, t s \exp (\pi(w+\lambda)(\mu)+\pi \overline{\lambda(z)})) .
$$

The projection $P \rightarrow V \times V^{*}$ induces a map $W_{\mathbb{Z}} \backslash P \rightarrow T \times T^{\vee}$. The action of $\mathbb{C}^{\times}$ on $P$ by acting on the third factor provides $W_{\mathbb{Z}} \backslash P$ with a structure of $\mathbb{C}^{\times}$-bundle over $T \times T^{\vee}$. Denote by $\mathcal{P}_{T}=\left(W_{\mathbb{Z}} \backslash P\right) \times \mathbb{C}$ the associated holomorphic line bundle. The structure of $P$ as a product space induces a canonical rigidification $\left.\mathcal{P}_{T}\right|_{\{0\} \times T^{\vee}}=\mathcal{O}_{\{0\} \times T^{\vee}}$.

Proposition 2.7. The line bundle $\mathcal{P}_{T}$ is a rigidified Poincaré line bundle.

Proof. From the explicit description of the cocycle equation (2.3) and of the action equation (2.7) we deduce that $\mathcal{P}_{T}$ is a Poincaré bundle.

The metric of the Poincaré bundle. The Poincaré bundle has a metric that is determined up to constant by the condition that its curvature form is invariant under translation. On a rigidified Poincaré bundle, with given rigidification $\left.\mathcal{P}_{T}\right|_{\{0\} \times T^{\vee}} \stackrel{\sim}{\rightarrow} \mathcal{O}_{\{0\} \times T^{\vee}}$, the constant is fixed by imposing the condition $\|1\|=1$. We now describe explicitly this metric.

Let $\widetilde{W}_{1}=W \ltimes \mathbb{C}_{1}$ with the product described before. Denote by $\mathcal{P}_{T}^{\times}$the Poincare bundle with the zero section deleted. Since $\mathcal{P}_{T}^{\times}=W_{\mathbb{Z}} \backslash P$, the invariant metric of $\mathcal{P}_{T}$ is described by the unique function $\|\cdot\|: P \rightarrow \mathbb{R}_{>0}$ satisfying the conditions

(1) (Norm condition) For $(z, w, s) \in P$, we have

$$
\|(z, w, s)\|=|s|\|(z, w, 1)\| .
$$

(2) (Invariance under $\widetilde{W}_{1}$ ) For $g \in \widetilde{W}_{1}$ and $x \in P$, we have

$$
\|g \cdot x\|=\|x\|
$$

(3) (Normalization) $\|(0,0,1)\|=1$.

Using the explicit description of the action given in equation (2.7), we have that

$$
(z, w, s)=(z, w, 1) \cdot(0,0, s \exp (-\pi w(z))),
$$

from which one easily derives that the previous conditions imply

$$
\|(z, w, s)\|^{2}=|s|^{2} \exp (-\pi(w(z)+\overline{w(z)})) .
$$


Holomorphic families of complex tori. Let $X$ be a complex manifold and $\mathcal{T} \rightarrow X$ a holomorphic family of dimension $g$ complex tori. This means that $\mathcal{T}$ is defined by a holomorphic vector bundle $\mathcal{V}$ of rank $g$ on $X$ and an integral local system $\Lambda \subset \mathcal{V}$ of rank $2 g$ such that, for each $s \in X$, the fiber $\Lambda_{s}$ is a lattice in $\mathcal{V}_{s}$ and the flat sections of $\Lambda$ are holomorphic sections of $\mathcal{V}$. Indeed $\Lambda$ is the local system $s \mapsto$ $H_{1}\left(\mathcal{T}_{s}, \mathbb{Z}\right)$ and $\mathcal{V}$ the holomorphic vector bundle $s \mapsto H_{1}\left(\mathcal{T}_{s}, \mathbb{C}\right) / F^{0} H_{1}\left(\mathcal{T}_{s}, \mathbb{C}\right)$.

We now want to give to the dual family of compact tori a holomorphic structure. That is, we want to construct a holomorphic family of compact tori $\mathcal{T}^{\vee}$ with a canonical identification $\left(\mathcal{T}^{\vee}\right)_{s}=\left(\mathcal{T}_{s}\right)^{\vee}$. This construction is not completely obvious because the vector spaces $\left(\mathcal{V}_{s}\right)^{*}$ vary antiholomorphically with $s$. We will use the lattice $\Lambda$ to define a holomorphic structure on this family of vector spaces.

Write $\mathcal{H}_{\mathbb{C}}=\Lambda \otimes \mathcal{O}_{X}$. It is a holomorphic vector bundle, with a holomorphic surjection $\mathcal{H}_{\mathbb{C}} \rightarrow \mathcal{V}$ and an integral structure that determines a complex conjugation in $\mathcal{H}_{\mathbb{C}}$. The kernel $\mathcal{F}^{0}=\operatorname{Ker}\left(\mathcal{H}_{\mathbb{C}} \rightarrow \mathcal{V}\right)$ is a holomorphic vector bundle. For every $s \in X$, the surjection $\mathcal{H}_{\mathbb{C}} \rightarrow \mathcal{V}$ allows us to identify $\overline{\mathcal{F}}_{s}$ with $\mathcal{V}_{s}$, hence $\mathcal{F}_{s}^{0}$ with $\overline{\mathcal{V}}_{s}$. Let $\Lambda^{\vee}$ be the dual local system to $\Lambda$. On the dual vector bundle $\mathcal{H}^{\vee}=\Lambda^{\vee} \otimes \mathcal{O}_{X}$ consider the orthogonal complement $\left(\mathcal{F}^{0}\right)^{\perp}$ to $\mathcal{F}^{0}$. Then $\left(\mathcal{F}^{0}\right)^{\perp}$ is isomorphic with the dual vector bundle $\mathcal{V}^{\vee}$. The quotient $\mathcal{H}^{\vee} /\left(\mathcal{F}^{0}\right)^{\perp}$ is a holomorphic vector bundle that we denote by $\mathcal{V}^{*}$. The identification $\mathcal{F}_{s}^{0}=\overline{\mathcal{V}}_{s}$ gives us the equality

$$
\left(\mathcal{V}^{*}\right)_{s}=\left(\mathcal{H}^{\vee} /\left(\mathcal{F}^{0}\right)^{\perp}\right)_{s}=\left(\mathcal{F}_{s}^{0}\right)^{\vee}=\left(\overline{\mathcal{V}}_{s}\right)^{\vee}=\left(\mathcal{V}_{s}\right)^{*},
$$

that explains the notation.

Then the dual family of tori is defined as

$$
\mathcal{T}^{\vee}=\mathcal{V}^{*} / \Lambda^{\vee}
$$

Let $U \subset X$ be a small enough open subset such that the restriction of $\mathcal{T}$ to $U$ is topologically trivial. Choose $s_{0} \in U$ and an integral basis

$$
(a, b)=\left(a_{1}, \ldots, a_{g}, b_{1}, \ldots, b_{g}\right)
$$

of $\Lambda_{s_{0}}$ such that $\left(a_{1}, \ldots, a_{g}\right)$ is a complex basis of $\mathcal{V}_{s_{0}}$. By abuse of notation, we denote by $a_{i}, b_{i}, i=1, \ldots, g$ the corresponding flat sections of $\Lambda$. We can see them as holomorphic sections of $\mathcal{H}_{\mathbb{C}}$ and we will also denote by $a_{i}, b_{i}$ their images in $\mathcal{V}$. After shrinking $U$ if necessary, we can assume that the sections $a_{i}$ form a frame of $\mathcal{V}$, thus we can write

$$
\left(b_{1}, \ldots, b_{g}\right)=\left(a_{1}, \ldots, a_{g}\right) \Omega
$$

for a holomorphic map $\Omega: U \rightarrow M_{g}(\mathbb{C})$. We call $\Omega$ the period matrix of the variation on the basis $(a, b)$. Note that condition equation $(2.9)$ is equivalent to 
saying that $\mathcal{F}^{0} \subset \mathcal{H}_{\mathbb{C}}$ is generated by the columns of the matrix

$$
\left(\begin{array}{c}
-\Omega \\
\mathrm{Id}
\end{array}\right) \text {. }
$$

Writing $\mathcal{H}_{\mathbb{R}}$ for the real vector subbundle of $\mathcal{H}_{\mathbb{C}}$ formed by sections that are invariant under complex conjugation, we have that $\mathcal{F}^{0} \cap \mathcal{H}_{\mathbb{R}}=0$. This implies that $\operatorname{Im} \Omega$ is nondegenerate. The complex basis $\left(a_{1}, \ldots, a_{g}\right)$ gives us an identification of $\left.\mathcal{V}\right|_{U}$ with the trivial vector bundle $\operatorname{Col}_{g}(\mathbb{C})$ and the basis $(a, b)$ identifies $\Lambda$ with the trivial local system $\operatorname{Col}_{g}(\mathbb{Z}) \oplus \operatorname{Col}_{g}(\mathbb{Z})$. With these identifications, the inclusion $\Lambda \rightarrow \mathcal{V}$ is given by

$$
\left(\mu_{1}, \mu_{2}\right) \mapsto \mu=\mu_{1}+\Omega \mu_{2} .
$$

Let now $\left(a^{*}, b^{*}\right)=\left(a_{1}^{*}, \ldots, a_{g}^{*}, b_{1}^{*}, \ldots, b_{g}^{*}\right)$ be the basis of $\Lambda_{s_{0}}^{\vee}$ dual to $(a, b)$. As before we extend the elements $a_{i}^{*}, b_{i}^{*}, i=1, \ldots, g$ to flat sections of $\Lambda$ over $U$. Then $b_{1}^{*}, \ldots, b_{g}^{*}$ is a frame of $\mathcal{V}^{*}$. One can check that, on $\mathcal{V}^{*}$, the equality

$$
\left(a_{1}^{*}, \ldots, a_{g}^{*}\right)=-\left(b_{1}^{*}, \ldots, b_{g}^{*}\right) \Omega^{t}
$$

holds. Thus if we identify $\mathcal{V}^{*}$ with the trivial vector bundle $\operatorname{Row}_{g}(\mathbb{C})$ using the basis $\left(b^{*}\right)$ and $\Lambda^{\vee}$ with the trivial local system $\operatorname{Row}_{g}(\mathbb{Z}) \oplus \operatorname{Row}_{g}(\mathbb{Z})$ using the basis $\left(a^{*}, b^{*}\right)$ we obtain that the inclusion $\Lambda^{\vee} \rightarrow \mathcal{V}^{*}$ is given by

$$
\left(\lambda_{1}, \lambda_{2}\right) \mapsto \lambda=-\lambda_{1} \Omega+\lambda_{2} .
$$

In the fixed bases, one can check that the pairing between $\mathcal{V}^{*}$ and $\mathcal{V}$ is given by

$$
w(z)=-w(\operatorname{Im} \Omega)^{-1} \bar{z},
$$

where $w \in \operatorname{Row}_{g}(\mathbb{C})$ and $z \in \operatorname{Col}_{g}(\mathbb{C})$, while the pairing between the lattice $\Lambda$ and its dual $\Lambda^{\vee}$ is given by

$$
\left\langle\left(\lambda_{1}, \lambda_{2}\right),\left(\mu_{1}, \mu_{2}\right)\right\rangle=\lambda_{1} \mu_{1}+\lambda_{2} \mu_{2},
$$

where $\lambda_{1}, \lambda_{2} \in \operatorname{Row}_{g}(\mathbb{Z})$ and $\mu_{1}, \mu_{2} \in \operatorname{Col}_{g}(\mathbb{Z})$. Clearly the pairing between $\Lambda$ and $\Lambda^{\vee}$ has integer values.

The cocycle $a_{\mathcal{P}}$ from equation (2.3) can now be written down explicitly as

$$
\begin{aligned}
& a_{\mathcal{P}}\left(\left(\mu_{1}, \mu_{2}\right),\left(\lambda_{1}, \lambda_{2}\right),(z, w)\right) \\
&= \exp \left(-\pi\left(\left(w-\lambda_{1} \Omega+\lambda_{2}\right)(\operatorname{Im} \Omega)^{-1}\left(\mu_{1}+\bar{\Omega} \mu_{2}\right)\right.\right. \\
&\left.\left.\quad+\left(-\lambda_{1} \bar{\Omega}+\lambda_{2}\right)(\operatorname{Im} \Omega)^{-1} z\right)\right),
\end{aligned}
$$

which is not holomorphic with respect to $\Omega$. Thus it does not give us on the nose a holomorphic Poincaré bundle in families. Nevertheless the construction of the Poincaré bundle can be given a holomorphic structure. 
Proposition 2.8. Let $X$ be a complex manifold and $\mathcal{T} \rightarrow X$ a holomorphic family of dimension $g$ complex tori. Let $v_{0}: X \rightarrow \mathcal{T} \underset{X}{\times} \mathcal{T}^{\vee}$ be the zero section. Then

(1) the fiberwise dual tori form a holomorphic family of complex tori $\mathcal{T}^{\vee} \rightarrow X$;

(2) on $\mathcal{T} \underset{X}{\times} \mathcal{T}^{\vee}$ there is a holomorphic line bundle $\mathcal{P}$, together with an isomorphism $v_{0}^{*} \mathcal{P} \stackrel{\sim}{\rightarrow} \mathcal{O}_{X}$, called the rigidified Poincaré bundle, which is unique up to a unique isomorphism, and is characterized by the property that for every point $p \in X$, the restriction $\left.\mathcal{P}\right|_{\mathcal{T}_{p} \times \mathcal{T}_{p}^{\vee}}$ is the rigidified Poincaré bundle of $\mathcal{T}_{p}$;

(3) there is a unique metric on $\mathcal{P}$ that induces the trivial metric on $v_{0}^{*} \mathcal{P}=\mathcal{O}_{X}$ and whose curvature is fiberwise translation invariant.

Proof. Fix an open subset $U \subset X$ as before. The dual family of tori $\mathcal{T}^{\vee}$ is holomorphic by definition.

In order to prove that the Poincaré bundle defines a holomorphic line bundle on the family we need to exhibit a new cocycle that is holomorphic in $z, w$ and $\Omega$ and that, for fixed $\Omega$, is equivalent to $a_{\mathcal{P}}$ holomorphically in $z$ and $w$. Write $\lambda=-\lambda_{1} \Omega+\lambda_{2}$ and $\mu=\mu_{1}+\Omega \mu_{2}$ as before with $\lambda_{1}, \lambda_{2} \in \operatorname{Row}_{g}(\mathbb{Z})$ and $\mu_{1}$, $\mu_{2} \in \operatorname{Col}_{g}(\mathbb{Z})$. Consider the cocycle

$$
b_{\mathcal{P}}((\lambda, \mu),(z, w))=\exp \left(2 \pi i\left(\left(w-\lambda_{1} \Omega+\lambda_{2}\right) \mu_{2}-\lambda_{1} z\right)\right)
$$

for $w \in \operatorname{Row}_{g}(\mathbb{C})$ and $z \in \operatorname{Col}_{g}(\mathbb{C})$. Then $b_{\mathcal{P}}$ is holomorphic in $z, w$, and $\Omega$. Consider also the function

$$
\psi(z, w)=\exp \left(-\pi w(\operatorname{Im} \Omega)^{-1} z\right)
$$

which is holomorphic in $z$ and $w$. Since

$$
b_{\mathcal{P}}((\mu, \lambda),(z, w))=a_{\mathcal{P}}((\mu, \lambda),(z, w)) \psi(z, w) \psi(z+\mu, w+\lambda)^{-1}
$$

we deduce that the cocycle $b_{\mathcal{P}}$ determines a line bundle that satisfies the properties stated in item (2.8) from the proposition over the open $U$. The uniqueness follows again from the seesaw principle. By the uniqueness, we can glue together the rigidified Poincaré bundles obtained in different open subsets $U$ to obtain a rigidified Poincaré bundle over $X$.

The fact that the invariant metric has invariant curvature fixes it up to a function on $X$ that is determined by the normalization condition. Thus if it exists, it is unique. Since the expression for the metric in equation (2.8) is smooth in $\Omega$ and 
the change of cocycle function in equation (2.14) is also smooth in $\Omega$ we obtain an invariant metric locally. Again the uniqueness implies that we can patch together the different local expressions.

REMARK 2.9. Since the cocycle $a_{\mathcal{P}}$ does not vary holomorphically in families, the frame for the Poincaré bundle used in equation (2.8) is not holomorphic in families. The cocycle $b_{\mathcal{P}}$ and the rigidification do determine a holomorphic frame of the Poincaré bundle over $X \times V \times V^{*}$. In this holomorphic frame the metric is given by

$$
\begin{aligned}
\|(z, w, s)\|^{2} & =|s|^{2} \exp (-\pi(w(z)+\overline{w(z)}))|\psi(z, w)|^{2} \\
& =|s|^{2} \exp \left(4 \pi \operatorname{Im}(w)(\operatorname{Im} \Omega)^{-1} \operatorname{Im}(z)\right),
\end{aligned}
$$

where $\psi$ is the function given in (2.14).

Abelian varieties. We now specialize to the case of polarized abelian varieties. A polarization on the torus $T=V / \Lambda$ is the datum of an antisymmetric nondegenerate bilinear form $E: \Lambda \times \Lambda \rightarrow \mathbb{Z}$ such that for all $v, w \in V$,

$$
E(i v, i w)=E(v, w), \quad-E(i v, v)>0, \text { for } v \neq 0 .
$$

Here we have extended $E$ by $\mathbb{R}$-bilinearly to $V=\Lambda \otimes \mathbb{R}$. Note that the standard convention in the literature on abelian varieties is to ask $E(i v, v)$ to be positive. But this convention is not compatible with the usual convention in the literature on Hodge Theory. We have changed the sign here to have compatible conventions for abelian varieties and for Hodge structures.

Since $E$ is antisymmetric and nondegenerate we can choose an integral basis $(a, b)$ such that the matrix of $E$ on $(a, b)$ is given by

$$
\left(\begin{array}{cc}
0 & \Delta \\
-\Delta & 0
\end{array}\right)
$$

where $\Delta$ is an integral diagonal matrix. We will call such basis a $\mathbb{Q}$-symplectic integral basis. From a $\mathbb{Q}$-symplectic integral basis $(a, b)$ we can construct a symplectic rational basis $\left(a \Delta^{-1}, b\right)$.

With the choice of a $\mathbb{Q}$-symplectic integral basis, the condition $E(i v, i w)=$ $E(v, w)$ is equivalent to the product matrix $\Delta \Omega$ being symmetric. Thus $\Omega^{t} \Delta=$ $\Delta \Omega$. The condition $-E(i v, v)>0$ is equivalent to $\Delta \operatorname{Im} \Omega$ being positive definite. This last condition is equivalent to that any of the symmetric matrices $(\operatorname{Im} \Omega)^{t} \Delta$, $\left((\operatorname{Im} \Omega)^{-1}\right)^{t} \Delta$ or $\Delta(\operatorname{Im} \Omega)^{-1}$ is positive definite.

Recall from (2.9) that $\Omega \in M_{g}(\mathbb{C})$ is determined by the relation $b=a \Omega$. The polarization $E$ defines a positive definite Hermitian form $H$ on $V$ given by

$$
H(v, w)=-E(i v, w)-i E(v, w),
$$


so that we recover the polarization $E$ as the restriction of $-\operatorname{Im}(H)$ to $\Lambda \times \Lambda$. In the basis $\left(a_{1}, \ldots, a_{g}\right)$ of $V$, the Hermitian form $H$ is given by $\Delta(\operatorname{Im} \Omega)^{-1}=$ $\left((\operatorname{Im} \Omega)^{-1}\right)^{t} \Delta$. That is, under the identification $V=\operatorname{Col}_{g}(\mathbb{C})$, we have

$$
H(v, w)=v^{t} \Delta(\operatorname{Im} \Omega)^{-1} \bar{w} .
$$

The polarization defines an isogeny $\lambda_{E}: T \rightarrow T^{\vee}$ that is given by the map $V \rightarrow V^{*}, v \mapsto H(v,-)$. Under the identification $V^{*}=\operatorname{Row}_{g}(\mathbb{C})$ given by the basis $\left(b^{*}\right)$, by equations (2.11) and (2.17), we deduce that $\lambda_{E}$ is given by

$$
\lambda_{E}(v)=-v^{t} \Delta .
$$

The fact that $\Delta \Omega$ is symmetric and $\Delta$ is integral implies that this map sends $\Lambda$ to $\Lambda^{\vee}$ defining an isogeny. The dual polarization $E^{\vee}$ on $V^{*}$ is given by the Hermitian form $H^{\vee}(e, f)=e(\operatorname{Im} \Omega)^{-1} \Delta^{-1} \bar{f}^{t}$ so that the map $V \rightarrow V^{*}$ is an isometry.

Consider now the composition of the diagonal map with the polarization map on the second factor (id, $\lambda_{E}$ ): $T \rightarrow T \times T^{\vee}$ and let $\mathcal{P}$ be the Poincaré bundle on $T \times T^{\vee}$. Then (id, $\left.\lambda_{E}\right)^{*} \mathcal{P}$ is an ample line bundle on $T$ whose first Chern class agrees with the given polarization of $T$.

THEOREM 2.10. The metric induced on the bundle $\left(\mathrm{id}, \lambda_{E}\right)^{*} \mathcal{P}$ is given by the function $\|\cdot\|: V \times \mathbb{C}^{\times} \rightarrow \mathbb{R}_{>0}$,

$$
\|(z, s)\|^{2}=|s|^{2} \exp \left(-4 \pi \operatorname{Im}(z)^{t} \Delta(\operatorname{Im} \Omega)^{-1} \operatorname{Im}(z)\right) .
$$

Proof. This follows from equations (2.15) and (2.18).

Hodge structures of type $(-1,0),(0,-1)$. Recall that a pure Hodge structure of type $(-1,0),(0,-1)$ is given by

(1) A finite rank $\mathbb{Z}$-module, $H_{\mathbb{Z}}$.

(2) A decreasing filtration $F^{\bullet}$ on $H_{\mathbb{C}} \stackrel{\text { def }}{=} H_{\mathbb{Z}} \otimes \mathbb{C}$ such that

$$
F^{-1} H_{\mathbb{C}}=H_{\mathbb{C}}, \quad F^{1} H_{\mathbb{C}}=0, \quad H_{\mathbb{C}}=F^{0} H_{\mathbb{C}} \oplus \overline{F^{0} H_{\mathbb{C}}} .
$$

A polarization of a Hodge structure of type $(-1,0),(0,-1)$ is a nondegenerate antisymmetric bilinear form $Q: H_{\mathbb{Z}} \otimes H_{\mathbb{Z}} \rightarrow \mathbb{Z}$ which, when extended to $H_{\mathbb{C}}$ by linearity, satisfies the 'Riemann bilinear relations'

(1) The subspace $F^{0} H_{\mathbb{C}}$ is isotropic.

(2) If $x \in F^{0} H_{\mathbb{C}}$, then $i Q(x, \bar{x})>0$. 
We will be interested only in torsion-free Hodge structures. We recall that the category of torsion-free Hodge structures of type $(-1,0),(0,-1)$ and the category of compact complex tori are equivalent; see [4, Exercise 1.5.10]. If $H=$ $\left(H_{\mathbb{Z}}, F^{\bullet}\right)$ is such a Hodge structure, we write $V=H_{\mathbb{C}} / F^{0}$ and $\pi: H_{\mathbb{C}} \rightarrow H_{\mathbb{C}} / F^{0}$ for the projection. Then $\Lambda \stackrel{\text { def }}{=} \pi\left(H_{\mathbb{Z}}\right)$ is a lattice in $V$, that defines a torus $T=V / \Lambda$. This torus is denoted by $J(H)$ and called the Jacobian of $H$.

Conversely, if $T$ is a complex torus, then $H_{1}(T, \mathbb{Z})$ is torsion-free and has a Hodge structure of type $(-1,0),(0,-1)$.

If $\left(H_{\mathbb{Z}}, F^{\bullet}\right)$ has a polarization $Q$ then, identifying $\Lambda$ with $H_{\mathbb{Z}}$ and writing $E=Q$, we obtain a polarization of $T$. We finish by verifying that, indeed $E$ is a polarization in the sense of complex tori. That $E$ is nondegenerate follows from the nondegeneracy of $Q$. Let $v, w \in V$, choose $\bar{x}, \bar{y} \in \overline{F^{0} H_{\mathbb{C}}}$ such that $\pi(\bar{x})=v$ and $\pi(\bar{y})=w$. Write $x, y$ for the complex conjugates of $\bar{x}$ and $\bar{y}$, respectively. Then $x+\bar{x} \in H_{\mathbb{Z}} \otimes \mathbb{R}$ and $\pi(x+\bar{x})=v$, while $i x-i \bar{x} \in H_{\mathbb{Z}} \otimes \mathbb{R}$ and $\pi(i x-i \bar{x})=-i v$. Thus by the first Riemann bilinear relation

$$
\begin{aligned}
E(i v, i w) & =Q(-i x+i \bar{x},-i y+i \bar{y})=Q(x, \bar{y})+Q(\bar{x}, y) \\
E(v, w) & =Q(x+\bar{x}, y+\bar{y})=Q(x, \bar{y})+Q(\bar{x}, y) .
\end{aligned}
$$

Thus $E(i v, i w)=E(v, w)$. Moreover, by the second bilinear relation

$$
H(v, v)=-E(i v, v)=-Q(-i x+i \bar{x}, x+\bar{x})=2 i Q(x, \bar{x})>0 .
$$

2.3. Nilpotent orbit theorem. The aim of this section is to formulate a version of the Nilpotent orbit theorem that allows us to deal with variations of mixed Hodge structures, in a setting with several variables. Such a Nilpotent orbit theorem is stated and proved in [19]. In order to formulate this theorem, we need quite a bit of background material and in particular define the notion of 'admissibility' for variations of mixed Hodge structures. Also we need to take a detailed look at the behavior of monodromy on the fibers of the underlying local systems. Most of the introductory material below is taken from [22, Section 14.4] and [19].

Variations of polarized mixed Hodge structures. Let $X$ be a complex manifold. A graded-polarized variation of mixed Hodge structures on $X$ is a local system $\boldsymbol{H} \rightarrow X$ of finitely generated abelian groups equipped with:

(1) A finite increasing filtration

$$
\boldsymbol{W}_{\bullet}: \quad 0 \subseteq \cdots \subseteq \boldsymbol{W}_{k} \subseteq \boldsymbol{W}_{k+1} \subseteq \cdots \subseteq \boldsymbol{H}_{\mathbb{Q}}
$$

of $\boldsymbol{H}_{\mathbb{Q}}=\boldsymbol{H} \otimes \mathbb{Q}$ by local subsystems, called the weight filtration. 
(2) A finite decreasing filtration

$$
\mathcal{F}^{\bullet}: \quad \boldsymbol{H}_{\mathbb{C}} \otimes \mathcal{O}_{X} \supseteq \cdots \supseteq \mathcal{F}^{p-1} \supseteq \mathcal{F}^{p} \supseteq \cdots \supseteq 0
$$

of the vector bundle $\mathcal{H}=\boldsymbol{H}_{\mathbb{C}} \otimes \mathcal{O}_{X}$ by holomorphic subbundles, called the Hodge filtration.

(3) For each $k \in \mathbb{Z}$ a nondegenerate bilinear form

$$
\boldsymbol{Q}_{k}: \operatorname{Gr}_{k}^{W}\left(\boldsymbol{H}_{\mathbb{Q}}\right) \otimes \operatorname{Gr}_{k}^{W}\left(\boldsymbol{H}_{\mathbb{Q}}\right) \rightarrow \mathbb{Q}_{X}
$$

of parity $(-1)^{k}$,

such that:

(1) For each $p \in \mathbb{Z}$ the Gauss-Manin connection $\nabla$ on $\mathcal{H}$ satisfies the 'Griffiths transversality condition' $\nabla \mathcal{F}^{p} \subseteq \Omega_{X}^{1} \otimes \mathcal{F}^{p-1}$,

(2) For each $k \in \mathbb{Z}$ the triple $\left(\mathrm{Gr}_{k}^{W}\left(\boldsymbol{H}_{\mathbb{Q}}\right), \mathcal{F}^{\bullet} \mathrm{Gr}_{k}^{W}(\mathcal{H}), \boldsymbol{Q}_{k}\right)$ is a variation of pure polarized rational Hodge structures of weight $k$. Here for each $p \in \mathbb{Z}$ we write $\mathcal{F}^{p} \mathrm{Gr}_{k}^{W}(\mathcal{H})$ for the image of $\mathcal{F}^{p} \mathcal{H} \cap \boldsymbol{W}_{k} \mathcal{H}$ in $\mathrm{Gr}_{k}^{W}\left(\boldsymbol{H}_{\mathbb{C}}\right)$ under the projection $\operatorname{map} \boldsymbol{W}_{k} \mathcal{H} \rightarrow \mathrm{Gr}_{k}^{W}\left(\boldsymbol{H}_{\mathbb{C}}\right)$.

A variation of polarized mixed Hodge structures will be called torsion-free if $\boldsymbol{H}$ is a local system of torsion-free abelian groups. A $\mathbb{Q}$-variation of polarized mixed Hodge structures is defined analogously with the difference that $\boldsymbol{H}$ is a local system of finite-dimensional $\mathbb{Q}$-vector spaces.

Period domains. If $\left(H, W_{\bullet}, F^{\bullet}\right)$ is a mixed Hodge structure, then $H_{\mathbb{C}}$ has a unique bigrading $I^{\bullet \bullet \bullet}$ such that

$$
F^{p} H_{\mathbb{C}}=\oplus_{r \geqslant p, s} I^{r, s}, \quad W_{k} H_{\mathbb{C}}=\oplus_{r+s \leqslant k} I^{r, s}, \quad I^{r, s}=\bar{I}^{s, r} \bmod \oplus_{p<r, q<s} I^{p, q} .
$$

The integers $h^{r, s}=\operatorname{dim} I^{r, s}$ are called the Hodge numbers of $\left(H, W_{\bullet}, F^{\bullet}\right)$.

Given a quadruple $\left(H, W_{\bullet}, Q_{k}, h\right)$ with $H$ a rational vector space, $W_{\bullet}$ an increasing filtration of $H, Q_{k}$ a collection of nondegenerate bilinear forms of parity $(-1)^{k}$ on $\mathrm{Gr}_{k}^{W}(H)$, and a partition of $\operatorname{dim}(H)$ into a sum of nonnegative integers $h=\left\{h^{r, s}\right\}$ satisfying the symmetry condition $h^{r, s}=h^{s, r}$, there exists a natural classifying space (also known as a period domain) $\mathcal{M}=\mathcal{M}(h)=\mathcal{M}(H$, $\left.W_{\bullet}, Q_{k}, h\right)$ of mixed Hodge structures $\left(W_{\bullet}, F^{\bullet}\right)$ on $H$ which are graded-polarized by $Q_{k}$.

We recall the construction of $\mathcal{M}$ from [19, Section 3]. Write

$$
f^{p}=\sum_{r \geqslant p, s} h^{r, s} \quad \text { and } \quad f_{k}^{p}=\sum_{r \geqslant p} h^{r, k-r}
$$


and let $\check{\mathcal{M}}$ be the set of all decreasing filtrations $F^{\bullet}$ of $H_{\mathbb{C}}$ satisfying

$$
\operatorname{dim}\left(F^{p}\right)=f^{p}, \quad \operatorname{dim}\left(F^{p} \mathrm{Gr}_{k}^{W}\right)=f_{k}^{p}, \quad \text { and } \quad Q_{k}\left(F^{p} \mathrm{Gr}_{k}^{W}, F^{k-p+1} \mathrm{Gr}_{k}^{W}\right)=0 .
$$

The group

$$
G_{\mathbb{C}}=\left\{g \in \operatorname{GL}\left(H_{\mathbb{C}}\right)^{W} \mid \operatorname{Gr}_{k}(g) \in \operatorname{Aut}_{\mathbb{C}}\left(Q_{k}\right)\right\}
$$

is a complex algebraic group that acts transitively on $\check{\mathcal{M}}$ giving to it a structure of complex manifold. The manifold $\check{\mathcal{M}}$ is usually called the 'compact dual' of $\mathcal{M}$ by analogy with the pure case, although in general it is not compact.

The period domain $\mathcal{M}$ is the subset of $\check{\mathcal{M}}$ formed by the filtrations $F^{\bullet}$ such that $\left(H, W_{\bullet}, F^{\bullet}, Q\right)$ is a polarized $\mathbb{Q}$-mixed Hodge structure. By [19, Lemma 3.9] $\mathcal{M}$ is an open subset of $\check{\mathcal{M}}$, hence it has an induced structure of complex manifold. By the same lemma, the group

$$
G_{P}=\left\{g \in \operatorname{GL}\left(H_{\mathbb{C}}\right)^{W} \mid \operatorname{Gr}_{k}(g) \in \operatorname{Aut}_{\mathbb{R}}\left(Q_{k}\right)\right\}
$$

acts transitively on $\mathcal{M}$. We also consider the group

$$
G_{\mathbb{R}}=\left\{g \in \operatorname{GL}\left(H_{\mathbb{R}}\right)^{W} \mid \operatorname{Gr}_{k}(g) \in \operatorname{Aut}_{\mathbb{R}}\left(Q_{k}\right)\right\} .
$$

Note that we have inclusions

$$
G_{\mathbb{R}} \subset G_{P} \subset G_{\mathbb{C}}
$$

REMARK 2.11. The group $G_{\mathbb{R}}$ acts transitively on the subset $\mathcal{M}_{\mathbb{R}}$ of filtrations defining a mixed Hodge structure that is split over $\mathbb{R}$. If the filtration $W$ has only two nontrivial weights that are adjacent, that is, if there is a $k$ such that

$$
0=W_{k-2} \subset W_{k-1} \subset W_{k}=H,
$$

then any mixed Hodge structure on $\mathcal{M}(H, W, Q, h)$ is split over $\mathbb{R}$. Therefore $\mathcal{M}_{\mathbb{R}}=\mathcal{M}$ and $G_{\mathbb{R}}$ acts transitively on $\mathcal{M}$. This will hold for the case of interest to us in Section 2.4.

Relative filtrations. Let $H$ be a rational vector space, equipped with a finite increasing filtration $W_{\text {. }}$. We let $N$ denote a nilpotent endomorphism of $H$, compatible with $W_{\bullet}$. We call an increasing filtration $M_{\bullet}$ of $H$ a weight filtration for $N$ relative to $W_{\bullet}$ if the two following conditions are satisfied:

(1) for each $i \in \mathbb{Z}$ we have $N M_{i} \subseteq M_{i-2}$,

(2) for each $k \in \mathbb{Z}$ and each $i \in \mathbb{N}$ we have that $N^{i}$ induces an isomorphism

$$
N^{i}: \mathrm{Gr}_{k+i}^{M} \mathrm{Gr}_{k}^{W} H \stackrel{\sim}{\rightarrow} \mathrm{Gr}_{k-i}^{M} \mathrm{Gr}_{k}^{W} H
$$

of vector spaces. 
It can be verified that if $H$ has a weight filtration for $N$ relative to $W_{\bullet}$, then it is unique. We call $N$ strict if $N(H) \cap W_{k}=N\left(W_{k}\right)$ for all $k \in \mathbb{Z}$. By [26, Proposition 2.16], if the filtration $W_{\bullet}$ has length two (in the sense that $H=W_{k}$ and $W_{k-2}=0$ for some $k$ ), and if $H$ has a weight filtration for $N$ relative to $W_{\bullet}$, then $N$ is strict.

Admissible variations of mixed Hodge structures. Now let $\left(\boldsymbol{H}, \boldsymbol{W}_{\bullet}, \mathcal{F}^{\bullet}, \boldsymbol{Q}_{k}\right)$ be a variation of graded-polarized mixed Hodge structures over the punctured unit disc $\Delta^{*}$. Let $s_{0} \in \Delta^{*}$ and $\left(H, W_{\bullet}, F^{\bullet}, Q_{k}\right)$ the fiber of $\left(\boldsymbol{H}, \boldsymbol{W}_{\bullet}, \mathcal{F}^{\bullet}, \boldsymbol{Q}_{k}\right)$ over $s_{0}$. Let $\gamma$ be a generator of the fundamental group $\pi_{1}\left(\Delta^{*}, s_{0}\right)$ and $T$ the monodromy operator defined by $\gamma$ acting on $H$. Since $\boldsymbol{W} \bullet$ is a filtration by local subsystems, the monodromy operator preserves $W_{\text {. }}$. The operator $T$ can be written as $T=$ $T_{s} T_{u}$, where $T_{s}$ is semisimple and $T_{u}$ is unipotent. The monodromy is said to be quasiunipotent if $T_{s}^{r}=$ Id for certain integer $r \geqslant 1$. We denote by $N=\log T_{u}$ the logarithm of the unipotent part of the monodromy. Clearly $N$ is nilpotent.

By [11, II Remarque 5.5] the vector bundle $\mathcal{H}=\boldsymbol{H} \otimes_{\mathbb{C}} \mathcal{O}_{\Delta^{*}}$ can be 'canonically' extended to a vector bundle $\tilde{\mathcal{H}}$ on the unit disk. Moreover, the subbundles $\mathcal{W}_{k}=$ $\boldsymbol{W}_{k} \otimes \mathcal{O}_{\Delta^{*}}$ also extend canonically to subbundles $\tilde{\mathcal{W}}_{k}$ of $\tilde{\mathcal{H}}$. These extensions are really canonical when the monodromy is unipotent. In case it is not, the extensions depend on the choice of a logarithm, but, as explained in [11, Section II 5] this choice can be made once and for all.

Following $[\mathbf{1 7}, 22]$ and [26] we call the variation $\left(\boldsymbol{H}, \boldsymbol{W}_{\bullet}, \mathcal{F}^{\bullet}, \boldsymbol{Q}_{k}\right)$ preadmissible if:

(1) the monodromy is quasiunipotent;

(2) the logarithm $N$ of the unipotent part of the monodromy has a weight filtration $M_{\bullet}\left(H, W_{\bullet}, N\right)$ relative to $W_{\bullet}$ on $H$;

(3) the subbundles $\mathcal{F}^{\bullet}$ of $\boldsymbol{H}$ extend to subbundles $\tilde{\mathcal{F}}^{\bullet}$ of $\tilde{\mathcal{H}}$ in such a way that the coherent sheaves $\operatorname{Gr}_{\tilde{\mathcal{F}}}^{p} \mathrm{Gr}_{k}^{\tilde{\mathcal{W}}}$ are locally free.

Assume now that $X$ is an open submanifold of a manifold $\bar{X}$, where $D=$ $\bar{X} \backslash X$ is a normal crossings divisor. Let $\left(\boldsymbol{H}, \boldsymbol{W}_{\bullet}, \mathcal{F}^{\bullet}, \boldsymbol{Q}_{k}\right)$ be a graded-polarized variation of mixed Hodge structures over the complex manifold $X$. We then call the variation $\left(\boldsymbol{H}, \boldsymbol{W}_{\bullet}, \mathcal{F}^{\bullet}, \boldsymbol{Q}_{k}\right)$ admissible if for every holomorphic map $\bar{f}: \Delta \rightarrow \bar{X}$ with $\bar{f}\left(\Delta^{*}\right) \subset X$, the variation $f^{*} \boldsymbol{H}$ on $\Delta^{*}$ is preadmissible. Here we denote by $f$ the restriction of $\bar{f}$ to $\Delta^{*}$.

In algebraic geometry, admissible variations of mixed Hodge structures come about as follows. Let $\pi: Y \rightarrow X$ be a morphism of complex algebraic varieties. Then there is a nonempty open subset $\iota: U \rightarrow X$ such that the constructible sheaf $\boldsymbol{H}=\iota^{*} \mathrm{R}^{i} \pi_{*} \mathbb{Z}_{Y}$ is a local system that has a canonical structure of 
admissible graded-polarized variation of mixed Hodge structures $\left(\boldsymbol{H}, \boldsymbol{W}_{\bullet}, \mathcal{F}^{\bullet}\right.$, $\boldsymbol{Q}_{k}$ ). Moreover, there is a finite étale map $g: \widetilde{U} \rightarrow U$ such that $g^{*} \boldsymbol{H}$ has unipotent monodromy.

In general, the usual cohomological operations like direct images or relative cohomology will produce mixed Hodge modules [24, 25] which are a generalization of the notion of admissible variations of polarizable mixed Hodge structures, where the local system $\boldsymbol{H}$ is replaced by a perverse sheaf of $\mathbb{Q}$-vector spaces. There is a criterion for when a mixed Hodge module is indeed an admissible variation of mixed Hodge structures: given a mixed Hodge module $\boldsymbol{H}$ (with a given polarization), if the underlying perverse sheaf is a local system, then $\boldsymbol{H}$ is an admissible $\mathbb{Q}$-variation of polarized mixed Hodge structures. See for instance [2] for a survey on mixed Hodge modules.

For admissible variations of mixed Hodge structures we have the following compatibility between the graded polarization and the monodromy. Let $\left(H, W_{\bullet}\right.$, $F^{\bullet}, Q_{k}$ ) be a reference fiber of the variation near the boundary divisor $D=\bar{X} \backslash X$ of the smooth algebraic variety $X$. We denote the local monodromy operators around the branches of $D$ by $T_{1}, \ldots, T_{m}$, and the corresponding logarithms of the unipotent part by $N_{1}, \ldots, N_{m}$. We denote by $\mathfrak{g}_{\mathbb{C}}$ the Lie algebra Lie $G_{\mathbb{C}}$ of the group $C_{\mathbb{C}}$ defined above. Then, in this generality, the $T_{i}$ belong to $G_{\mathbb{C}}$, and the $N_{i}$ belong to $\mathfrak{g}_{\mathbb{C}}$, for each $i=1, \ldots, m$. The $\mathbb{R}_{>0}$-span $\mathcal{C}$ of the local monodromy logarithms $N_{i}$ inside $\mathfrak{g}_{\mathbb{C}}$ is called the open monodromy cone of the reference fiber $\left(H, W_{\bullet}, F^{\bullet}, Q_{k}\right)$. Each element of $\mathcal{C}$ is nilpotent, and it can be proved that the relative weight filtration $M_{\bullet}$ of $\left(H, W_{\bullet}\right)$ is constant on $\mathcal{C}$.

The period map. To an admissible graded-polarized variation of mixed Hodge structures $\left(\boldsymbol{H}, \boldsymbol{W}_{\bullet}, \mathcal{F}^{\bullet}, \boldsymbol{Q}_{k}\right)$ over $X=\left(\Delta^{*}\right)^{k} \times \Delta^{n-k}$ we can associate a period map, as follows. Let $\mathcal{M}=\mathcal{M}(h)$ be the period domain associated to $\left(H, W_{\bullet}, Q_{k}\right)$ and set $G_{\mathbb{C}}$ as above. Let $\Gamma \subset G_{\mathbb{C}}$ be the image of the monodromy representation $\rho: \pi_{1}\left(X, x_{0}\right) \rightarrow G_{\mathbb{C}}$. The period map $\phi: X \rightarrow \Gamma \backslash \mathcal{M}$ is the map that associates to $x \in X$ the Hodge filtration of $\boldsymbol{H}_{x}$. The period map is holomorphic.

Let $\mathbb{H} \subset \mathbb{C}$ be the upper half plane. Let $e: \mathbb{H}^{k} \rightarrow\left(\Delta^{*}\right)^{k}$ be the uniformization map given by $\left(z_{1}, \ldots, z_{k}\right) \mapsto\left(\exp \left(2 \pi i z_{1}\right), \ldots, \exp \left(2 \pi i z_{k}\right)\right)$. Then along $e$ the period map $\phi$ lifts to a map $\tilde{\phi}: \mathbb{H}^{k} \times \Delta^{n-k} \rightarrow \mathcal{M}$. In other words, we have the following commutative diagram

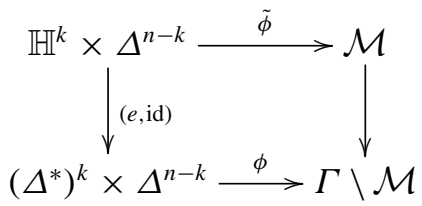

where the right hand arrow is the canonical projection. As $N_{i} \in \operatorname{Lie} G_{\mathbb{C}}$ we find 
$\exp \left(\sum_{i=1}^{k} z_{i} N_{i}\right) \in G_{\mathbb{C}}$ for all $z_{1}, \ldots, z_{k} \in \mathbb{H}$. Let $\tilde{\psi}: \mathbb{H}^{k} \times \Delta^{n-k} \rightarrow \check{\mathcal{M}}$ be the map given by

$$
\tilde{\psi}\left(z_{1}, \ldots, z_{k}, q_{k+1}, \ldots, q_{n}\right)=\exp \left(-\sum_{i=1}^{k} z_{i} N_{i}\right) \tilde{\phi}\left(z_{1}, \ldots, z_{k}, q_{k+1}, \ldots, q_{n}\right) .
$$

Then $\tilde{\psi}$ descends to an 'untwisted' period map $\psi:\left(\Delta^{*}\right)^{k} \times \Delta^{n-k} \rightarrow \breve{\mathcal{M}}$, fitting in a commutative diagram

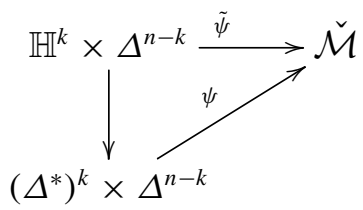

Note that, importantly, the map $\psi$ takes values in the compact dual $\check{\mathcal{M}}$, and not in a quotient of it.

Nilpotent orbit theorem. The following result is the starting point of Pearlstein's Nilpotent orbit theorem (see [19, Section 6]) for admissible graded-polarized variations of mixed Hodge structures and is enough for showing the estimates we need.

THEOREM 2.12 (Pearlstein). Let $\left(\boldsymbol{H}, \boldsymbol{W}_{\bullet}, \mathcal{F}^{\bullet}, \boldsymbol{Q}_{k}\right)$ be an admissible gradedpolarized variation of mixed Hodge structures over $X=\left(\Delta^{*}\right)^{k} \times \Delta^{n-k}$. Then the untwisted period map $\psi$ extends to a holomorphic map $\psi: \Delta^{n} \rightarrow \breve{\mathcal{M}}$.

2.4. Families of pointed polarized abelian varieties. Period vectors. Assume that $\left(H, F^{\bullet}, Q\right)$ is a polarized pure Hodge structure of weight -1 , torsionfree, of type $(-1,0),(0,-1)$, and of rank $2 g$. Recall that given a $\mathbb{Q}$-symplectic integral basis $\left(a_{1}, \ldots, a_{g}, b_{1}, \ldots, b_{g}\right)$ of $(H, Q)$, there exists a unique basis $\left(w_{1}, \ldots, w_{g}\right)$ of $F^{0} H_{\mathbb{C}}$ determined by demanding that $w_{i}=-\sum_{j=1}^{g} \Omega_{i j} a_{j}+b_{i}$ for some (period) matrix $\Omega \in M_{g}(\mathbb{C})$ (see equation (2.9)). We call this new basis the associated normalized basis. As we have seen, the Riemann bilinear relations imply that $\Omega^{t} \Delta=\Delta \Omega$ and $\Delta \operatorname{Im} \Omega>0$.

Assume an extension

$$
0 \rightarrow H \rightarrow H^{\prime} \rightarrow \mathbb{Z}(0) \rightarrow 0
$$

in the category of mixed Hodge structures is given. Then $H^{\prime}$ has weight filtration

$$
W_{\bullet}: \quad 0 \subset W_{-1}=H_{\mathbb{Q}} \subset W_{0}=H_{\mathbb{Q}}^{\prime} .
$$


Taking $F^{0}(-)_{\mathbb{C}}$ in $(2.21)$ yields the extension

$$
0 \rightarrow F^{0} H_{\mathbb{C}} \rightarrow F^{0} H_{\mathbb{C}}^{\prime} \rightarrow \mathbb{C} \rightarrow 0
$$

of $\mathbb{C}$-vector spaces. As can be readily checked, for each $a_{0} \in H^{\prime}$ that lifts the canonical generator of $\mathbb{Z}(0)$ in (2.21) there exists a unique $w_{0} \in F^{0} H_{\mathbb{C}}^{\prime}$ such that $w_{0} \in a_{0}+\mathbb{C}-\operatorname{span}\left(a_{1}, \ldots, a_{g}\right)$. Given such a lift $a_{0}$, we let $\delta_{H^{\prime}}=\left(\delta_{1}\right.$, $\left.\ldots, \delta_{g}\right)^{t} \in \operatorname{Col}_{g}(\mathbb{C})$ be the coordinate vector determined by the identity $w_{0}=$ $a_{0}+\sum_{j=1}^{g} \delta_{j} a_{j}$. We call $\delta_{H^{\prime}}$ the period vector of the mixed Hodge structure $\left(H^{\prime}\right.$, $\left.F^{\bullet}, W_{\bullet}\right)$ on the basis $\left(a_{0}, a_{1}, \ldots, a_{g}, b_{1}, \ldots, b_{g}\right)$ of the $\mathbb{Z}$-module $H^{\prime}$. It can be verified that replacing $a_{0}$ by some element from $a_{0}+H$ changes $\delta$ by an element of $\mathbb{Z}^{g}+\Omega \mathbb{Z}^{g}$. The resulting map $\operatorname{Ext}_{\mathrm{MHS}}^{1}(\mathbb{Z}(0), H) \rightarrow \mathbb{C}^{g} /\left(\mathbb{Z}^{g}+\Omega \mathbb{Z}^{g}\right)$ is finite, and gives $\operatorname{Ext}_{\mathrm{MHS}}^{1}(\mathbb{Z}(0), H)$ a canonical structure of complex torus.

Let $A=J(H)$ be the Jacobian of $H$. Thus $A$ is a polarized complex abelian variety of dimension $g$ with $H=H_{1}(A)$. Let $v \in A$, and write $H(v)$ for the relative homology group $H_{1}(A,\{0, v\})$. There is an extension of mixed Hodge structures

$$
0 \rightarrow H \rightarrow H(v) \rightarrow \mathbb{Z}(0) \rightarrow 0
$$

canonically associated to $(A, v)$. Here $\mathbb{Z}(0)$ is to be identified with the reduced homology group $\tilde{H}_{0}(\{0, v\})$. The map $A \rightarrow \operatorname{Ext}_{\mathrm{MHS}}^{1}(\mathbb{Z}(0), H)$ given by sending $v$ to the extension $H(v)$ is a bijection, compatible with the structure of complex torus on left and right hand side.

The period map of a family of pointed polarized abelian varieties. Let $X$ be a smooth complex variety with an open immersion $X \subset \bar{X}$ into a smooth complex algebraic variety, with $D=\bar{X} \backslash X$ a normal crossings divisor. Let $\left(\boldsymbol{H}, \mathcal{F}^{\bullet}, \boldsymbol{Q}_{k}\right)$ be a variation of polarized torsion-free pure Hodge structures of weight -1 and type $(-1,0),(0,-1)$ over $X$. We note that such a pure polarized variation is necessarily admissible. Write $Y=J(\boldsymbol{H})$ and let $\pi: Y \rightarrow X$ be the associated analytic family of polarized abelian varieties over $X$, with polarization $\lambda: Y \rightarrow Y^{\vee}$.

We will now work locally complex analytically. Thus we will suppose that $\bar{X}$ is the polydisk $\Delta^{n}$, and $D$ is the divisor given by the equation $q_{1} \cdots q_{k}=0$, so that $X=\left(\Delta^{*}\right)^{k} \times \Delta^{n-k}$. We further assume that all local monodromy operators $T_{1}, \ldots, T_{k}$ about the various branches determined by $q_{1}, \ldots, q_{k}$ are unipotent (for instance, this is the case if the family extends as a semiabelian scheme $\bar{Y} \rightarrow \bar{X}$ ). Let $g$ be the relative dimension of $Y \rightarrow X$. Also, we will henceforth usually suppress the polarization from our notation.

Let $\left(H, F^{\bullet}\right)$ be a reference fiber of $\boldsymbol{H}$ near the origin. Let $N$ be any element of the open monodromy cone of $H$. Then we have $N^{2}=0$ and the filtration 
associated to $N$ simply reads

$$
0 \subset M_{-2} \subset M_{-1} \subset M_{0}=H_{\mathbb{Q}}
$$

with $M_{-2}=\operatorname{Im} N$ and $M_{-1}=\operatorname{Ker} N$. Since, in this case the group $G_{\mathbb{R}}$ acts transitively on $\mathcal{M}$, the operator $N$ belongs to the Lie algebra of $G_{\mathbb{R}}$, thus there exist a $\mathbb{Q}$-symplectic integral basis $\left(a_{1}, \ldots, a_{g}, b_{1}, \ldots, b_{g}\right)$ of $(H, Q)$ and a nonnegative integer $r \leqslant g$ such that:

(1) $M_{-2}=\operatorname{span}\left(a_{1}, \ldots, a_{r}\right)$;

(2) $M_{-1}=\operatorname{span}\left(a_{1}, \ldots, a_{g}, b_{r+1}, \ldots, b_{g}\right)$.

In particular, $\left(\bar{a}_{r+1}, \ldots, \bar{a}_{g}, \bar{b}_{r+1}, \ldots, \bar{b}_{g}\right)$ is a $\mathbb{Q}$-symplectic integral basis of the pure polarized Hodge structure $\mathrm{Gr}_{-1}^{M} H$ of type $(-1,0),(0,-1)$. Clearly, with respect to this basis, each local monodromy operator $N_{j}$ has the form

$$
N_{j}=\left(\begin{array}{c|c}
0 & A_{j}^{\prime} \\
\hline 0 & 0
\end{array}\right) .
$$

Let $\Delta$ be the matrix associated to the polarization as in equation (2.16). Each $A_{j}^{\prime}$ is integral and the $g$-by- $g$ matrices $A_{j} \stackrel{\text { def }}{=} \Delta A_{j}^{\prime}$ are symmetric and positive semidefinite. Moreover, the left upper $r$-by- $r$ block of $A_{j}$ is positive definite. See [9] for more details in the above construction.

To avoid the appearance of the polarization matrix $\Delta$ and thus to simplify the notation we will sometimes replace the $\mathbb{Q}$-symplectic integral basis $(a, b)$ by the symplectic $\mathbb{Q}$-basis $\left(a \Delta^{-1}, b\right)$. In this new basis each local monodromy operator $N_{j}$ has the form

$$
N_{j}=\left(\begin{array}{c|c}
0 & A_{j} \\
\hline 0 & 0
\end{array}\right) .
$$

On this new basis we can realize the period domain associated to $H$ as the usual Siegel's upper half space $\mathbb{H}_{g}$ of rank $g$. We have $G_{\mathbb{R}}=\operatorname{Sp}(2 g, \mathbb{R})$, and the action on $\mathbb{H}_{g}$ is given by the usual prescription

$$
\left(\begin{array}{l|l}
A \mid B \\
\hline C & D
\end{array}\right) \cdot M=(A M+B)(C M+D)^{-1}, \quad\left(\frac{A \mid B}{C \mid D}\right) \in \operatorname{Sp}(2 g, \mathbb{R}), \quad M \in \mathbb{H}_{g} .
$$

In this representation the period map $\Omega: X \rightarrow \Gamma \backslash \mathbb{H}_{g}$ is made explicit by associating to each $x \in X$ the matrix $\Omega(x)=\Delta \Omega_{Y_{x}}$, where $\Omega_{Y_{x}}$ is the period matrix of the fiber $Y_{x}$ on the chosen $\mathbb{Q}$-symplectic integral basis of $H$. Here $\Gamma$ is the image of the monodromy representation into $\operatorname{Sp}(2 g, \mathbb{R})$. In the new basis, the monodromy representation sends the local monodromy operator $T_{j}$ to the matrix

$$
\left(\begin{array}{c|c}
1 & A_{j} \\
\hline 0 & 1
\end{array}\right) \in \operatorname{Sp}(2 g, \mathbb{R}) \text {. }
$$


We will now extend this picture to include an admissible normal function $v$.

DEFINITION 2.13. Let $U \subset X$ be a nonempty open subset. An admissible normal function of $Y$ on $U$ is a holomorphic section $v:\left.U \rightarrow Y\right|_{U}$ such that the associated graded-polarized mixed Hodge structures $\boldsymbol{H}_{x}(\nu), x \in U$ form an admissible variation over $U$.

Let $v$ be an admissible normal function of $Y$ on $X$. Varying $x \in X$ we thus find an extension

$$
0 \rightarrow \boldsymbol{H} \rightarrow \boldsymbol{H}(v) \rightarrow \mathbb{Z}(0) \rightarrow 0
$$

of admissible variations of graded-polarized mixed Hodge structure. The weight filtration of this variation looks like

$$
W_{\bullet}: \quad 0 \subset \boldsymbol{W}_{-1}=\boldsymbol{H}_{\mathbb{Q}} \subset \boldsymbol{W}_{0}=\boldsymbol{H}(v)_{\mathbb{Q}},
$$

so that $\mathrm{Gr}_{-1}^{\boldsymbol{W}} \boldsymbol{H}(v)_{\mathbb{Q}}=\boldsymbol{H}_{\mathbb{Q}}$ and $\mathrm{Gr}_{0}^{\boldsymbol{W}} \boldsymbol{H}(v)=\mathbb{Q}(0)$. We denote the Hodge filtration of $\boldsymbol{H}(v)$ by $\mathcal{F}^{\bullet}$. We start by taking a reference fiber $H(v)$ of $\boldsymbol{H}(v)$ and augmenting our chosen $\mathbb{Q}$-symplectic integral basis of $H$ by an $a_{0} \in H(v)$ lifting the canonical generator of $\mathbb{Z}(0)$.

Since by assumption, $\boldsymbol{H}(v)$ is an admissible variation of polarized mixed Hodge structures, the relative weight filtration $M^{\prime}$. on our reference fiber $H(v)$ exists. Let $N^{\prime}$ be an element of the open monodromy cone of $H(v)$ such that $N=\left.N^{\prime}\right|_{H}$. We will now proceed to determine the matrix shape of $N^{\prime}$ on the basis $\left(a_{0}, a \Delta^{-1}, b\right)$ of $H(v)$. As $N^{\prime 2}=0$, the filtration associated to $N^{\prime}$ on $H(v)$ is

$$
L_{\bullet}: \quad 0 \subset L_{-1} \subset L_{0} \subset L_{1}=H(v)_{\mathbb{Q}},
$$

with $L_{-1}=\operatorname{Im}\left(N^{\prime}\right), L_{0}=\operatorname{Ker}\left(N^{\prime}\right)$. As the monodromy action on $\mathrm{Gr}_{0}^{W}=\mathbb{Q}(0)$ is trivial, we have that $\operatorname{Im}\left(N^{\prime}\right) \subset H_{\mathbb{Q}}$, so that $N^{\prime-1} H_{\mathbb{Q}}=H(v)_{\mathbb{Q}}$ (here by $N^{\prime-1} H_{\mathbb{Q}}$ we denote the inverse image of $H_{\mathbb{Q}}$ under $N^{\prime}$ ). As $W_{\bullet}$ has length two, and as by admissibility the weight filtration of $N^{\prime}$ relative to $W_{\bullet}$ exists, as we noted above it follows that $N^{\prime}$ is strict. Explicitly, we have that $H(v)_{\mathbb{Q}}=N^{\prime-1} H_{\mathbb{Q}}=H_{\mathbb{Q}}+$ $\operatorname{Ker}\left(N^{\prime}\right)$. The equality $H(v)_{\mathbb{Q}}=H_{\mathbb{Q}}+\operatorname{Ker}\left(N^{\prime}\right)$ implies that $\operatorname{Ker}\left(N^{\prime}\right) \supsetneqq \operatorname{Ker}(N)$ and hence that $\operatorname{Im}\left(N^{\prime}\right)=\operatorname{Im}(N)$.

The period domain associated to $\left(H(v), W_{\bullet}\right)$ can be realized as $\mathbb{C}^{g} \times \mathbb{H}_{g}$. The group $G_{\mathbb{R}}$ in this case is

$$
G_{\mathbb{R}}=\left\{\left(\begin{array}{c|c|c}
1 & 0 & 0 \\
\hline m & A & B \\
\hline n & C & D
\end{array}\right): m, n \in \mathbb{R}^{g},\left(\begin{array}{c|c}
A \mid B \\
\hline C & D
\end{array}\right) \in \operatorname{Sp}(2 g, \mathbb{R})\right\}
$$


The action of $G_{\mathbb{R}}$ on $\mathbb{C}^{g} \times \mathbb{H}_{g}$ is given by

$$
\left(\begin{array}{c|c|c}
1 & 0 & 0 \\
\hline m & A & B \\
\hline n & C & D
\end{array}\right)(v, M)=\left(v+m+M n,(A M+B)(C M+D)^{-1}\right), v \in \mathbb{C}^{g}, M \in \mathbb{H}_{g} .
$$

Varying $x \in X=\left(\Delta^{*}\right)^{k} \times \Delta^{n-k}$ and then taking $F^{0}$ we obtain a period map associated to the variation $\boldsymbol{H}(v)$

$$
(\delta, \Omega): X \rightarrow \Gamma \backslash\left(\mathbb{C}^{g} \times \mathbb{H}_{g}\right)
$$

that is given by

$$
(\delta(x), \Omega(x))=\left(\Delta \delta_{H(v(x))}, \Delta \Omega_{Y_{x}}\right) .
$$

We denote by

$$
(\tilde{\delta}, \tilde{\Omega}): \mathbb{H}^{k} \times \Delta^{n-k} \rightarrow \mathbb{C}^{g} \times \mathbb{H}_{g}
$$

the lift of the period map along the map (e, id): $\mathbb{H}^{k} \times \Delta^{n-k} \rightarrow X$, where we recall that we denote by $e: \mathbb{H}^{k} \rightarrow\left(\Delta^{*}\right)^{k}$ the map

$$
e\left(z_{1}, \ldots, z_{k}\right)=\left(\exp \left(2 \pi i z_{1}\right), \ldots, \exp \left(2 \pi i z_{k}\right)\right)
$$

THEOREM 2.14. There exist a holomorphic map $\psi: \Delta^{n} \rightarrow S_{g}(\mathbb{C})$, a holomorphic map $\alpha: \Delta^{n} \rightarrow \mathbb{C}^{g}$, and vectors $c_{1}, \ldots, c_{k} \in \mathbb{Q}^{g}$ with $\Delta^{-1} A_{j} c_{j} \in \mathbb{Z}^{g}$ for $j=1$, $\ldots, k$ such that for $(z, t) \in \mathbb{H}^{k} \times \Delta^{n-k}$ with $e(z)$ sufficiently close to zero the equalities

$$
\tilde{\Omega}(z, t)=\sum_{j=1}^{k} z_{j} A_{j}+\psi(e(z), t), \quad \tilde{\delta}(z, t)=\sum_{j=1}^{k} z_{j} A_{j} c_{j}+\alpha(e(z), t)
$$

hold in $S_{g}(\mathbb{C})$ respectively $\mathbb{C}^{g}$.

Proof. Let $N_{j}$ denote the local monodromy operator of $H$ around the branch of $D$ determined by $q_{j}=0$. We have

$$
\exp \left(z_{j} N_{j}\right)=T_{j}^{z_{j}}=\left(\begin{array}{c|c}
1 & z_{j} A_{j} \\
\hline 0 & 1
\end{array}\right)
$$

and hence $\exp \left(z_{j} N_{j}\right) \cdot M=z_{j} A_{j}+M$ for each $M \in \mathbb{H}_{g}, z_{j} \in U$, and $j=1$, $\ldots, k$ (here $U$ is an open subset of $\mathbb{H}$ consisting of points with sufficiently large imaginary part). Denote by $\mathbb{P}_{g}$ the compact dual of $\mathbb{H}_{g}$. The untwisted period map 
$\psi: \Delta^{n} \rightarrow \mathbb{P}_{g}$ obtained by Theorem 2.12 extending $\exp \left(-\sum_{j=1}^{k} z_{j} N_{j}\right) . \tilde{\Omega}(z, t)$ factors through $S_{g}(\mathbb{C}) \subset \mathbb{P}_{g}$. We obtain the equalities

$$
\tilde{\Omega}(z, t)=\exp \left(\sum_{j=1}^{k} z_{j} N_{j}\right) \cdot \psi(e(z), t)=\sum_{j=1}^{k} z_{j} A_{j}+\psi(e(z), t)
$$

in $S_{g}(\mathbb{C})$.

Let $N_{j}^{\prime}$ denote the local monodromy operator of $H(v)$ around the branch of $D$ determined by $q_{j}=0$. The equality $\operatorname{Im}\left(N_{j}^{\prime}\right)=\operatorname{Im}\left(\left.N_{j}^{\prime}\right|_{H_{\mathbb{Q}}}\right)$ on $H(\nu)_{\mathbb{Q}}$ that follows from our above considerations shows that $N_{j}^{\prime}$ has a matrix

$$
\left(\begin{array}{c|c|c}
0 & 0 & 0 \\
\hline \Delta^{-1} A_{j} c_{j} & 0 & \Delta^{-1} A_{j} \\
\hline 0 & 0 & 0
\end{array}\right)
$$

on the integral basis $\left(a_{0}, a_{1}, \ldots, a_{g}, b_{1}, \ldots, b_{g}\right)$, for some $c_{j} \in \mathbb{Q}^{g}$. Since the monodromy is integral in such basis, we deduce that $\Delta^{-1} A_{j} c_{j}$ has to be integral. In the $\mathbb{Q}$-basis $\left(a_{0}, a \Delta^{-1}, b\right)$, the matrix of $N_{j}^{\prime}$ is

$$
\left(\begin{array}{c|c|c}
0 & 0 & 0 \\
\hline A_{j} c_{j} & 0 & A_{j} \\
\hline 0 & 0 & 0
\end{array}\right)
$$

Then for $(v, M) \in \mathbb{C}^{g} \times \mathbb{H}_{g}$ and $z_{j} \in U$ we have $\exp \left(z_{j} N_{j}^{\prime}\right) .(v, M)=(v+$ $\left.z_{j} A_{j} c_{j}, M+z_{j} A_{j}\right)$. Let $(\alpha, \psi): \Delta^{n} \rightarrow \mathbb{C}^{g} \times \mathbb{P}_{g}$ denote the untwisted period map. We find the equalities

$$
\tilde{\delta}(z, t)=\exp \left(\sum_{j=1}^{k} z_{j} N_{j}^{\prime}\right) \cdot \alpha(e(z), t)=\sum_{j=1}^{k} z_{j} A_{j} c_{j}+\alpha(e(z), t)
$$

in $\mathbb{C}^{g}$.

The norm of a section. Denote by $\mathcal{P}$ the Poincaré bundle on $Y \times{ }_{X} Y^{\vee}$ with its canonical $C^{\infty}$ Hermitian metric as described in Section 2.2. Given two admissible normal functions $v, \mu: X \rightarrow Y$ we will denote

$$
\mathcal{P}_{\nu, \mu}=(\nu, \lambda \mu)^{*} \mathcal{P}, \quad \mathcal{P}_{\nu}=\mathcal{P}_{v, v},
$$

where $\lambda: Y \rightarrow Y^{\vee}$ is the isogeny provided by the polarization. We are interested in studying the singularities of the metric of $\mathcal{P}_{\nu, \mu}$ when we approach the boundary of $X$. 
Consider the five maps

$$
m, p_{1,3}, p_{1,4}, p_{2,3}, p_{2,4}: Y \times_{X} Y \times_{X} Y^{\vee} \times_{X} Y^{\vee} \longrightarrow Y \times_{X} Y^{\vee},
$$

where $m(x, y, z, t)=(x+y, z+t)$ and $p_{i, j}$ is the projection over the factors $i, j$. Then we have a canonical isomorphism

$$
m^{*} \mathcal{P} \stackrel{\sim}{\rightarrow} p_{1,3}^{*} \mathcal{P} \otimes p_{1,4}^{*} \mathcal{P} \otimes p_{2,3}^{*} \mathcal{P} \otimes p_{2,4}^{*} \mathcal{P}
$$

of holomorphic line bundles over $Y \times_{X} Y \times_{X} Y^{\vee} \times_{X} Y^{\vee}$, in other words, the Poincaré bundle is a biextension on $Y \times_{X} Y^{\vee}$ in the sense of [12, Exposé VII]. The explicit description of the cocycle $b_{\mathcal{P}}$ in equation (2.13) and of the metric of the Poincare bundle in Remark 2.9 shows that the canonical isomorphism (2.22) is in fact an isometry for the canonical induced metrics on left and right hand side. We obtain in particular

LEMMA 2.15. Let $v_{1}, v_{2}, \mu_{1}, \mu_{2}$ be holomorphic sections of the family $Y \rightarrow X$. Then we have a canonical isometry

$$
\begin{aligned}
& \left(v_{1}+v_{2}, \lambda\left(\mu_{1}+\mu_{2}\right)\right)^{*} \mathcal{P} \\
& \quad \stackrel{\sim}{\rightarrow}\left(v_{1}, \lambda \mu_{1}\right)^{*} \mathcal{P} \otimes\left(v_{1}, \lambda \mu_{2}\right)^{*} \mathcal{P} \otimes\left(v_{2}, \lambda \mu_{1}\right)^{*} \mathcal{P} \otimes\left(v_{2}, \lambda \mu_{2}\right)^{*} \mathcal{P}
\end{aligned}
$$

of Hermitian line bundles on $X$.

Let $f: Y \times_{X} Y^{\vee} \rightarrow Y \times_{X} Y^{\vee}$ be the map given by $(x, \ell) \mapsto\left(\lambda^{\vee}(\ell), \lambda(x)\right)$. Then we have a canonical isometry $f^{*} \mathcal{P} \stackrel{\sim}{\rightarrow} \mathcal{P}^{\otimes d}$, where $d=\operatorname{det} \Delta$ is the degree of the polarization $\lambda$. This leads to canonical isometries

$$
\mathcal{P}_{\nu, \mu}^{\otimes d} \stackrel{\sim}{\rightarrow} \mathcal{P}_{\mu, \nu}^{\otimes d} \quad \text { and } \quad \mathcal{P}_{\nu, \mu}^{\otimes 2 d} \stackrel{\sim}{\rightarrow} \mathcal{P}_{\nu+\mu}^{\otimes d} \otimes \mathcal{P}_{\nu}^{\otimes(-d)} \otimes \mathcal{P}_{\mu}^{\otimes(-d)}
$$

Hence, in order to study the singularities of the metric on $\mathcal{P}_{\nu, \mu}$ it suffices to study the singularities of the metric on $\mathcal{P}_{\nu}, \mathcal{P}_{\mu}$ and $\mathcal{P}_{\nu+\mu}$. In particular, for the purpose of proving our main results, it suffices to focus on the diagonal cases $\mathcal{P}_{\nu}$. Let $v$ be an admissible normal function of the family $Y \rightarrow X$. Let $s$ be a section on $X$ of $\mathcal{P}_{v}$. Interpreting the Poincaré bundle as parametrizing biextension variations of mixed Hodge structures (see [13]) we can canonically associate to the section $s$ a biextension variation of mixed Hodge structures over $X$. We say that the section $s$ is admissible if this variation is admissible.

Let $x_{0}$ be a point of $\bar{X}$. The purpose of the present section is to give an asymptotic expansion of the logarithm of the norm of an admissible section of $\mathcal{P}_{v}$ near $x_{0}$. From equation (2.19) it follows that it suffices to give asymptotic 
expansions of the period matrix of the family $Y \rightarrow X$, and of the period vector (see below) associated to $v$.

We use now Theorem 2.14 to obtain an expression of the norm of the section $s$. Let $\|-\|$ denote the canonical metric on $\mathcal{P}_{v}=v^{*} \mathcal{P}$. Continuing the notation from Theorem 2.14, let $a=2 \pi \operatorname{Im} \alpha$ and $B=2 \pi \operatorname{Im} \psi$. For $j=1, \ldots, k$ let $x_{j}=-\log \left|t_{j}\right|$.

COROLlARY 2.16. For every admissible section s of $\mathcal{P}_{\nu}$ on $\left(\Delta^{*}\right)^{k} \times \Delta^{n-k}$ there exists a meromorphic function $h$ on $\Delta^{n}$ which is holomorphic on $\left(\Delta^{*}\right)^{k} \times \Delta^{n-k}$, such that the identity

$$
-\log \|s\|=-\log |h|+\left(\sum_{j=1}^{k} x_{j} A_{j} c_{j}+a\right)^{t}\left(\sum_{j=1}^{k} x_{j} A_{j}+B\right)^{-1}\left(\sum_{j=1}^{k} x_{j} A_{j} c_{j}+a\right)
$$

holds on $\left(\Delta^{*}\right)^{k} \times \Delta^{n-k}$.

Proof. The vector $z$ and the matrix $\Omega$ in Theorem 2.10 are expressed in the integral basis $(a, b)$, while $\delta(x)$ and $\Omega(x)$ are expressed in the $\mathbb{Q}$-basis $\left(a \Delta^{-1}\right.$, $b)$. Writing $z=\Delta^{-1} \delta(x)$ and $\Omega=\Delta^{-1} \Omega(x)$, we obtain that

$$
-\log \|s(x)\|=-\log |h(x)|+2 \pi(\operatorname{Im} \delta(x))^{t}(\operatorname{Im} \Omega(x))^{-1}(\operatorname{Im} \delta(x))
$$

for a suitable meromorphic function $h$ on $\Delta^{n}$ which is holomorphic on $\left(\Delta^{*}\right)^{k} \times \Delta^{n-k}$. Note that, even though $\Omega(x), \delta(x)$ are multivalued, their imaginary parts are single valued. From Theorem 2.14 we obtain, noting that $\operatorname{Im} z_{j}=-1 / 2 \pi \log \left|t_{j}\right|$,

$$
\begin{aligned}
& \operatorname{Im} \Omega(x)=-\frac{1}{2 \pi} \sum_{j=1}^{k} A_{j} \log \left|t_{j}\right|+\operatorname{Im} \psi, \\
& \operatorname{Im} \delta(x)=-\frac{1}{2 \pi} \sum_{j=1}^{k} A_{j} c_{j} \log \left|t_{j}\right|+\operatorname{Im} \alpha .
\end{aligned}
$$

Combining we find equation (2.23).

\section{Normlike functions}

The purpose of this section is to carry out a systematic study of the functions

$$
\varphi=\left(\sum_{j=1}^{k} x_{j} A_{j} c_{j}+a\right)^{t}\left(\sum_{j=1}^{k} x_{j} A_{j}+B\right)^{-1}\left(\sum_{j=1}^{k} x_{j} A_{j} c_{j}+a\right)
$$


that appear on the right hand side of the equality in Corollary 2.16. We call such functions normlike functions.

Let $f: \mathbb{R}^{n} \rightarrow \mathbb{R}$ be a function. The recession function of $f$ is the function

$$
\operatorname{rec}(f)(x)=\lim _{\lambda \rightarrow+\infty} \frac{1}{\lambda} f(\lambda x) .
$$

If the recession function exists, it is homogeneous of weight one, in the sense that

$$
\operatorname{rec}(f)(\mu x)=\mu \operatorname{rec}(f)(x) \text { for } \mu \in \mathbb{R}_{>0} .
$$

We show that normlike functions $\varphi$ have a well-defined recession function $\operatorname{rec}(\varphi)$ with respect to the variables $x_{j}$, and we are able to calculate $\operatorname{rec}(\varphi)$ explicitly. In our main technical lemma Theorem 3.2 we give bounds for the difference $\varphi-\operatorname{rec}(\varphi)$ and, in the case where $k=1$, for the first- and secondorder derivatives of $\varphi-\operatorname{rec}(\varphi)$. The bound on the difference will be key to the proof of our first main result Theorem 1.1, the bounds on the derivatives will be used in our proof of Theorem 1.3. In Section 3.4 we prove, among other things, that the recession functions $\operatorname{rec}(\varphi)$ are convex. This will lead to the effectivity statement in Theorem 1.5.

3.1. Some definitions. Recall that we have denoted by $M_{r}(\mathbb{R})$ the space of $r$ by- $r$ matrices with real coefficients, by $S_{r}^{+}(\mathbb{R}) \subset M_{r}(\mathbb{R})$ the cone of symmetric positive semidefinite real matrices inside $M_{r}(\mathbb{R})$, and by $S_{r}^{++}(\mathbb{R}) \subset S_{r}^{+}(\mathbb{R})$ the cone of symmetric positive definite real matrices.

LEMMA 3.1. Let $N_{1}, \ldots, N_{k}$ be a finite set of positive semidefinite symmetric real g-by-g matrices such that $N_{1}+\cdots+N_{k}$ has rank $r$. Then there exists an orthogonal matrix $u \in O_{g}(\mathbb{R})$ such that, upon writing $M_{i}=u^{t} N_{i} u$ for $i=1, \ldots$, $k$, we have

$$
M_{i}=\left(\begin{array}{c|c}
M_{i}^{\prime} & 0 \\
\hline 0 & 0
\end{array}\right),
$$

with all $M_{i}^{\prime} \in S_{r}^{+}(\mathbb{R})$ and $\sum M_{i}^{\prime} \in S_{r}^{++}(\mathbb{R})$.

Proof. It will be convenient to use the language of bilinear forms. If $Q$ is a symmetric positive semidefinite bilinear form on $\mathbb{R}^{g}$ and $f_{1}, \ldots, f_{g}$ is a basis of $\mathbb{R}^{g}$ such that $Q\left(f_{\alpha}, f_{\alpha}\right)=0$ for $\alpha=r+1, \ldots, g$, then $Q\left(f_{\alpha}, f_{\beta}\right)=0$ for $\beta=1, \ldots, g$ and $\alpha=r+1, \ldots, g$. Indeed, for all $\lambda \in \mathbb{R}$ we have $Q\left(\lambda f_{\alpha}-f_{\beta}\right.$, $\left.\lambda f_{\alpha}-f_{\beta}\right) \geqslant 0$, that is

$$
-2 \lambda Q\left(f_{\alpha}, f_{\beta}\right)+Q\left(f_{\beta}, f_{\beta}\right) \geqslant 0 .
$$

Since this inequality is satisfied for all $\lambda$ we deduce that $Q\left(f_{\alpha}, f_{\beta}\right)=0$. 
Let $N=N_{1}+\cdots+N_{k}$, and denote by $Q$ the symmetric positive semidefinite bilinear form that $N$ defines on the standard basis $\left(e_{1}, \ldots, e_{g}\right)$ of $\mathbb{R}^{g}$. Note that $Q$ has rank $r$. By the spectral theorem, upon replacing the basis $\left(e_{1}, \ldots, e_{g}\right)$ of $\mathbb{R}^{g}$ by $\left(f_{1}, \ldots, f_{g}\right)=\left(e_{1}, \ldots, e_{g}\right) u$ for some orthogonal matrix $u$ we can assume that the expression of $Q$ in the basis $\left(f_{1}, \ldots, f_{g}\right)$ is

$$
M=\left(\begin{array}{l|l}
A & 0 \\
\hline 0 & 0
\end{array}\right),
$$

with $A \in S_{r}^{+}(\mathbb{R})$ invertible and diagonal. In particular, $Q\left(f_{\alpha}, f_{\alpha}\right)=0$ for $\alpha=$ $r+1, \ldots, g$. For $i=1, \ldots, k$ let $Q_{i}$ denote the symmetric positive semidefinite bilinear form that $N_{i}$ defines on the standard basis $\left(e_{1}, \ldots, e_{g}\right)$ of $\mathbb{R}^{g}$. Note that $Q=Q_{1}+\cdots+Q_{k}$. Since all the $Q_{i}$ are positive semidefinite, we deduce that $Q_{i}\left(f_{\alpha}, f_{\alpha}\right)=0$ for $i=1, \ldots, k$. Note that $M_{i}=u^{t} N_{i} u$ is the expression of $Q_{i}$ in the basis $\left(f_{1}, \ldots, f_{g}\right)$. By the previous discussion we have

$$
M_{i}=\left(\begin{array}{c|c}
M_{i}^{\prime} & 0 \\
\hline 0 & 0
\end{array}\right)
$$

with $M_{i}^{\prime} \in S_{r}^{+}(\mathbb{R})$ and $\sum M_{i}^{\prime}=A \in S_{r}^{++}(\mathbb{R})$, proving the lemma.

Suppose we are given the following data:

- three integers $k \geqslant 0, m \geqslant 0, g \geqslant 0$;

- a real number $\kappa \geqslant 0$;

- a compact subset $K \subseteq \mathbb{R}^{m}$;

- matrices $A_{1}, \ldots, A_{k} \in S_{g}^{+}(\mathbb{R})$ all of rank $\geqslant 1$;

- vectors $c_{1}, \ldots, c_{k} \in \mathbb{R}^{g}$;

- functions $a: K \rightarrow \mathbb{R}^{g}$ and $B: K \rightarrow S_{g}(\mathbb{R})$ which are restrictions of smooth functions on some open neighborhood of $K$;

such that for all $\left(x_{1}, \ldots, x_{k}, \lambda\right) \in \mathbb{R}_{>\kappa}^{k} \times K$, we have that

$$
P\left(x_{1}, \ldots, x_{k}, \lambda\right) \stackrel{\text { def }}{=} \sum_{i=1}^{k} x_{i} A_{i}+B(\lambda)>0 .
$$

Note that if $g=0$, then necessarily $k=0$.

To these data we associate a smooth function $\varphi: \mathbb{R}_{>\kappa}^{k} \times K \rightarrow \mathbb{R}$ by 


$$
\begin{aligned}
\varphi\left(x_{1}, \ldots, x_{k}, \lambda\right)= & \left(\sum_{i=1}^{k} x_{i} A_{i} c_{i}+a(\lambda)\right)^{t}\left(\sum_{i=1}^{k} x_{i} A_{i}+B(\lambda)\right)^{-1} \\
& \times\left(\sum_{i=1}^{k} x_{i} A_{i} c_{i}+a(\lambda)\right) .
\end{aligned}
$$

By condition (3.1), the function $\varphi$ is well defined and its values are nonnegative. We call $\varphi$ the normlike function associated to the 4-tuple $\left(\left(A_{i}\right),\left(c_{i}\right), a, B\right)$. We call the natural number $k$ the dimension of $\varphi$. Write $r=\operatorname{rk} \sum_{i=1}^{k} x_{i} A_{i}$ for some (hence all) $\left(x_{1}, \ldots, x_{k}\right) \in \mathbb{R}_{>\kappa}^{k}$. Note that $r \geqslant 1$ if $k>0$.

Let $u \in O_{g}(\mathbb{R})$. Replacing the vector $c_{i}$ by $u^{-1} c_{i}, a$ by $u^{-1} a$, the matrix $B$ by $u^{t} B u$ and $A_{i}$ by $u^{t} A_{i} u$ one checks that the function $\varphi$ remains unchanged. By Lemma 3.1 we can thus restrict to considering normlike functions where the $A_{i}$ have the shape

$$
A_{i}=\left(\begin{array}{c|c}
A_{i}^{\prime} & 0_{r, g-r} \\
\hline 0_{g-r, r} & 0_{g-r, g-r}
\end{array}\right),
$$

with each $A_{i}^{\prime} \in S_{r}^{+}(\mathbb{R})$ and such that $\sum x_{i} A_{i}^{\prime} \in S_{r}^{++}(\mathbb{R})$ for all $\left(x_{1}, \ldots, x_{k}\right) \in \mathbb{R}_{>\kappa}^{k}$ (hence for all $\left.\left(x_{1}, \ldots, x_{k}\right) \in \mathbb{R}_{>0}^{k}\right)$.

From now on we assume that the matrices $A_{i}$ indeed have this shape. We write

$$
c_{i}=\left(\frac{c_{i}^{\prime}}{\star_{g-r}}\right), \quad a=\left(\frac{a_{1}}{a_{2}}\right), \quad \text { and } \quad B=\left(\begin{array}{c|c}
B_{11} & B_{12} \\
\hline B_{21} & B_{22},
\end{array}\right)
$$

where $c_{i}^{\prime}$ and $a_{1}$ have size $r$, and $B_{11}$ is an $r$-by- $r$ matrix. The second block of the vector $c_{i}$ is marked with an asterisk because the function $\varphi$ is independent of its value. Condition (3.1) implies that $B_{22}(\lambda)$ is positive definite for all $\lambda \in K$, and the symmetry of $B$ implies that $B_{21}=B_{12}^{t}$.

We define another smooth function $f: \mathbb{R}_{>\kappa}^{k} \times K \rightarrow \mathbb{R}$ by

$$
f\left(x_{1}, \ldots, x_{k}, \lambda\right)=\left(\sum_{i=1}^{k} x_{i} A_{i}^{\prime} c_{i}^{\prime}\right)^{t}\left(\sum_{i=1}^{k} x_{i} A_{i}^{\prime}\right)^{-1}\left(\sum_{i=1}^{k} x_{i} A_{i}^{\prime} c_{i}^{\prime}\right) .
$$

This function $f$ is well defined as $\sum_{i=1}^{k} x_{i} A_{i}^{\prime}$ is positive definite on $\mathbb{R}_{>0}^{k}$. The function $f$ depends trivially on $\lambda$ and is clearly homogeneous of degree 1 in the $x_{i}$, and so defines a smooth function $\mathbb{R}_{>0}^{k} \rightarrow \mathbb{R}$, which we also call $f$. Again, the values of $f$ are nonnegative. By convention, if $k=0$, the function $f$ is zero.

Finally, the 'recession' of $\varphi$ is defined as the pointwise limit

$$
\begin{aligned}
\operatorname{rec}(\varphi): \quad \mathbb{R}_{>\kappa}^{k} \times K & \rightarrow \mathbb{R} \\
\left(x_{1}, \ldots, x_{k}, \lambda\right) & \mapsto \lim _{\mu \rightarrow \infty} \frac{1}{\mu} \varphi\left(\mu x_{1}, \ldots, \mu x_{k}, \lambda\right),
\end{aligned}
$$

if it exists. Again, if $k=0$, then $\operatorname{rec}(\varphi)=0$. 
3.2. Statement of the technical lemma. We can now state the 'main technical lemma':

THEOREM 3.2. In the notation of the previous section, write $\varphi_{0}=\varphi-f$. Note that $\varphi_{0}$ is a smooth function on $\mathbb{R}_{>\kappa} \times K$. Then

(1) the function $\left|\varphi_{0}\right|$ is bounded on $\mathbb{R}_{>\kappa^{\prime}}^{k} \times K$ for some $\kappa^{\prime} \geqslant \kappa$. The recession of $\varphi$ exists and is equal to $f$. In particular, $\operatorname{rec}(\varphi)$ is independent of the parameter $\lambda$;

(2) the function $f$ is bounded on the open simplex $\Delta^{0}=\left\{\left(x_{1}, \ldots, x_{k}\right) \in \mathbb{R}_{>0}^{k}\right.$ : $\left.\sum_{i=1}^{k} x_{i}=1\right\}$;

(3) when $k=1$,

(a) the function $\varphi_{0}: \mathbb{R}_{>_{\kappa}} \times K \rightarrow \mathbb{R}$ extends continuously to a function from $\overline{\mathbb{R}_{>\kappa}} \times K$ to $\mathbb{R}$, where by $\overline{\mathbb{R}_{>\kappa}}$ we denote $\mathbb{R}_{>\kappa} \sqcup\{\infty\}$ with the natural topology;

(b) the derivatives of $\varphi_{0}$ satisfy the estimates

$$
\frac{\partial \varphi_{0}}{\partial x_{1}}=O\left(x_{1}^{-2}\right) \quad \text { and } \quad \frac{\partial^{2} \varphi_{0}}{\partial x_{1}^{2}}=O\left(x_{1}^{-3}\right),
$$

as $x_{1} \rightarrow \infty$, where the implicit constant is uniform in $K$.

EXAMPLE 3.3. When $k>1$, in general we cannot extend $\varphi_{0}$ to a continuous function on ${\overline{\mathbb{R}_{>\kappa}}}^{k} \times K$ as the following example shows. Put $g=1, k=2, m=0$, $A_{1}=A_{2}=1, c_{1}=1, c_{2}=2, B=0, \kappa=1$ and $a=1$. Then

$$
\varphi_{0}=\varphi-f=\frac{2\left(x_{1}+2 x_{2}\right)+1}{x_{1}+x_{2}} .
$$

The sequences $\{(n, n)\}_{n \geqslant 1}$ and $\{(n, 2 n)\}_{n \geqslant 1}$ converge, when $n \rightarrow \infty$, to the point $(\infty, \infty) \in{\overline{\mathbb{R}_{>1}}}^{2}$. Nevertheless

$$
\lim _{n \rightarrow \infty} \varphi_{0}(n, n)=3, \quad \lim _{n \rightarrow \infty} \varphi_{0}(n, 2 n)=\frac{10}{3},
$$

showing that $\varphi_{0}$ cannot be continuously extended to $\overline{\mathbb{R}}_{>1}^{2}$.

Before starting the proof of Theorem 3.2 we recall a few easy statements related to Schur complements and inverting a symmetric block matrix. For a symmetric block matrix

$$
M=\left(\frac{A \mid B}{B^{t} \mid C}\right)
$$


with $C$ invertible we call $A-B C^{-1} B^{t}$ the Schur complement of the block $C$ in $M$. We have a product decomposition

$$
M=\left(\begin{array}{c|c}
A & B \\
\hline B^{t} & C
\end{array}\right)=\left(\begin{array}{c|c}
1 & B C^{-1} \\
\hline 0 & 1
\end{array}\right)\left(\begin{array}{c|c|c}
A-B C^{-1} B^{t} & 0 \\
\hline 0 & C
\end{array}\right)\left(\begin{array}{c|c}
1 & 0 \\
\hline C^{-1} B^{t} & 1
\end{array}\right) .
$$

In particular, $M$ is invertible if and only if $A-B C^{-1} B^{t}$ is invertible, and if these conditions are satisfied we have

$$
M^{-1}=\left(\begin{array}{c|c}
\left(A-B C^{-1} B^{t}\right)^{-1} & -\left(A-B C^{-1} B^{t}\right)^{-1} B C^{-1} \\
\hline-C^{-1} B^{t}\left(A-B C^{-1} B^{t}\right)^{-1} & C^{-1}+C^{-1} B^{t}\left(A-B C^{-1} B^{t}\right)^{-1} B C^{-1}
\end{array}\right) .
$$

Also, if $M$ is positive semidefinite, then so is the Schur complement $A-B C^{-1} B^{t}$.

3.3. Proof of the technical lemma. First we observe that, if $k=0$, then $\varphi$ is a continuous function on a compact set, hence is bounded. Moreover, the function $f$ is zero. Thus the statements are trivially true and we are reduced to the case $k>0$ and hence $g>0$.

Assume that we have already shown that $|\varphi-f|$ is bounded on $\mathbb{R}_{>\kappa^{\prime}}^{k} \times K$. Then, for each $\left(x_{1}, \ldots, x_{k}, \lambda\right) \in \mathbb{R}_{>\kappa^{\prime}}^{k} \times K$ we have

$$
\lim _{\mu \rightarrow \infty} \frac{1}{\mu} \varphi\left(\mu x_{1}, \ldots, \mu x_{k}, \lambda\right)=\lim _{\mu \rightarrow \infty} \frac{1}{\mu} f\left(\mu x_{1}, \ldots, \mu x_{k}\right) .
$$

The latter limit exists and is equal to $f\left(x_{1}, \ldots, x_{k}\right)$ by weight-one homogeneity of $f$. Thus the recession function of $\varphi$ exists and agrees with $f$. In consequence, in order to prove Theorem 3.2(1) and (2) we only need to show the boundedness of $|\varphi-f|$ and of $f$ on the required subsets.

We next show that we can assume a simplifying hypothesis.

DEFINITION 3.4. We say that the set of symmetric positive semidefinite matrices $A_{1}, \ldots, A_{k}$ satisfies the flag condition if $\operatorname{Ker}\left(A_{i}\right) \subseteq \operatorname{Ker}\left(A_{i+1}\right)$, for $i=1, \ldots$, $k-1$.

Consider the subset

$$
U=\left\{0<x_{1} \leqslant x_{2} \leqslant \cdots \leqslant x_{k}\right\} \subset \mathbb{R}_{>0}^{k} .
$$

Since

$$
\mathbb{R}_{>\kappa}^{k}=\bigcup_{\sigma \in \mathfrak{S}_{k}}\left(\sigma^{-1} U \cap \mathbb{R}_{>\kappa}^{k}\right)
$$


and

$$
\Delta^{0}=\bigcup_{\sigma \in \mathfrak{S}_{k}}\left(\sigma^{-1} U \cap \Delta^{0}\right),
$$

by symmetry it is enough to prove the boundedness of $|\varphi-f|$ in $U \cap \mathbb{R}_{>\kappa}^{k}$ and of $f$ in $U \cap \Delta^{0}$. Writing $y_{1}=x_{1}, y_{i}=x_{i}-x_{i-1}$ for $i=2, \ldots, k$ we find that $x_{i}=\sum_{j=1}^{i} y_{j}$ and that $U \cap \mathbb{R}_{>\kappa}^{k}$ is parametrized by the set $y_{1}>\kappa, y_{2}, \ldots, y_{k} \geqslant 0$.

Note that

$$
\sum_{i=1}^{k} x_{i} A_{i}=\sum_{i=1}^{k} y_{i} \sum_{j=i}^{k} A_{j} \quad \text { and } \quad \sum_{i=1}^{k} x_{i} A_{i} c_{i}=\sum_{i=1}^{k} y_{i} \sum_{j=i}^{k} A_{j} c_{j} .
$$

Lemma 3.5. Writing $\tilde{A}_{i}=\sum_{j=i}^{k} A_{j}$ we have that $\operatorname{Ker} \tilde{A}_{i} \subseteq \operatorname{Ker} \tilde{A}_{i+1}$. Moreover we have $\operatorname{Im}\left(\tilde{A}_{i}\right)=\sum_{j=i}^{k} \operatorname{Im}\left(A_{j}\right)$.

Proof. We first observe that, if $A$ is a symmetric positive semidefinite real matrix, then $A x=0$ if and only if $x^{t} A x=0$. Indeed, clearly $A x=0$ implies $x^{t} A x=0$. Conversely, assume that $x^{t} A x=0$ and let $y$ be any vector. Then, for all $\lambda \in \mathbb{R}$,

$$
0 \leqslant(y+\lambda x)^{t} A(y+\lambda x)=y^{t} A y+2 \lambda y^{t} A x
$$

which implies that $y^{t} A x=0$. Therefore $A x=0$.

We show that this observation implies that $\operatorname{Ker} \tilde{A}_{i}=\bigcap_{j=i}^{k} \operatorname{Ker} A_{j}$. We have $x \in \operatorname{Ker} \tilde{A}_{i}$ if and only if

$$
0=x^{t} \tilde{A}_{i} x=\sum_{j=i}^{k} x^{t} A_{j} x
$$

Since the matrices $A_{j}$ are positive semidefinite this implies that $x^{t} A_{j} x=0, j=i$, $\ldots, k$. Therefore $x \in \bigcap_{j=i}^{k} \operatorname{Ker} A_{j}$. The converse is clear. As a result

$$
\operatorname{Ker} \tilde{A}_{i}=\bigcap_{j=i}^{k} \operatorname{Ker} A_{j} \subseteq \bigcap_{j=i+1}^{k} \operatorname{Ker} A_{j}=\operatorname{Ker} \tilde{A}_{i+1}
$$

Since, for a symmetric positive semidefinite matrix $A$, the image $\operatorname{Im}(A)$ is the orthogonal complement of $\operatorname{Ker}(A)$ we deduce

$$
\operatorname{Im}\left(\tilde{A}_{i}\right)=\operatorname{Ker}\left(\tilde{A}_{i}\right)^{\perp}=\left(\bigcap_{j=i}^{k} \operatorname{Ker}\left(A_{j}\right)\right)^{\perp}=\sum_{j=i}^{k} \operatorname{Ker}\left(A_{j}\right)^{\perp}=\sum_{j=i}^{k} \operatorname{Im}\left(A_{j}\right) .
$$

This proves the lemma. 
It follows from the Lemma that there exist vectors $\tilde{c}_{i} \in \mathbb{R}^{g}$ such that

$$
\sum_{j=i}^{k} A_{j} c_{j}=\tilde{A}_{i} \tilde{c}_{i}
$$

Replacing $A_{i}$ by $\tilde{A}_{i}, x_{i}$ by $y_{i}$ and $c_{i}$ by $\tilde{c}_{i}$ we are reduced to proving the boundedness of $|\varphi-f|$ on $\mathbb{R}_{>\kappa} \times \mathbb{R}_{\geqslant 0}^{k-1} \times K$ and of $f$ on the set

$$
\left\{\left(x_{1}, \ldots, x_{k}\right) \in \mathbb{R}_{\geqslant 0}^{k}: x_{1}>0, x_{i} \geqslant 0 \text { for all } i>1, \sum_{i=1}^{k}(k-i+1) x_{i}=1\right\}
$$

under the extra hypothesis that the matrices $A_{1}, \ldots, A_{k}$ satisfy the flag condition from Definition 3.4. Clearly, by the homogeneity of $f$ it is enough to prove the boundedness of $f$ on the set

$$
H=\left\{\left(x_{1}, \ldots, x_{k}\right) \in \mathbb{R}_{\geqslant 0}^{k}: x_{1}>0, x_{i} \geqslant 0 \text { for all } i>1, \sum_{i=1}^{k} x_{i}=1\right\} .
$$

From now on we assume the flag condition and we write $r_{i}=\operatorname{rk}\left(A_{i}\right)$. Then $r=r_{1} \geqslant \cdots \geqslant r_{k} \geqslant 1$. Thanks to the flag condition, we can assume furthermore that the basis of $\mathbb{R}^{g}$ has been chosen in such a way that

$$
A_{i}^{\prime}=\left(\begin{array}{c|c}
A_{i}^{\prime \prime} & 0 \\
\hline 0 & 0
\end{array}\right)
$$

with $A_{i}^{\prime \prime} \in S_{r_{i}}^{++}(\mathbb{R})$.

The following is our main estimate.

LEMMA 3.6. There exists a constant $c$ such that for all $1 \leqslant \alpha, \beta \leqslant r$ and all $\left(x_{1}, \ldots, x_{k}\right) \in \mathbb{R}_{>0} \times \mathbb{R}_{\geqslant 0}^{k-1}$ we have the following bound on the $\alpha$, $\beta$-entry in the inverse of the r-by-r matrix $\sum_{i=1}^{k} x_{i} A_{i}^{\prime}$ :

$$
\left|\left(\sum_{i=1}^{k} x_{i} A_{i}^{\prime}\right)_{\alpha \beta}^{-1}\right| \leqslant \frac{c}{\sum_{j: r_{j} \geqslant \min (\alpha, \beta)} x_{j}} \leqslant \frac{c}{x_{1}} .
$$

Proof. This follows immediately from two intermediate results:

Claim 3.6.1. There exists a constant $c_{1}>0$ such that for all $\left(x_{1}, \ldots, x_{k}\right) \in$ $\mathbb{R}_{>0} \times \mathbb{R}_{\geqslant 0}^{k-1}$ we have the bound

$$
\operatorname{det}\left(\sum_{i=1}^{k} x_{i} A_{i}^{\prime}\right) \geqslant c_{1} \prod_{j=1}^{r} \sum_{i: r_{i} \geqslant j} x_{i}>0 .
$$


To prove this claim, define the $r$-by- $r$ matrix

$$
J_{i}=\left(\begin{array}{c|c}
\mathrm{Id}_{r_{i}} & 0 \\
\hline 0 & 0
\end{array}\right) .
$$

Since $A_{i}^{\prime \prime}$ is positive definite, there exists $\epsilon>0$ such that for all $i$, we have that $A_{i}^{\prime}-\epsilon J_{i}$ is positive semidefinite. Then

$$
\sum_{i} x_{i} A_{i}^{\prime}=\sum_{i} x_{i}\left(A_{i}^{\prime}-\epsilon J_{i}\right)+\sum_{i} x_{i} \epsilon J_{i}
$$

The proof of the following lemma is left to the reader.

LeMma 3.7. Let $A, B$ be positive semidefinite symmetric $r \times r$ matrices. Then $\operatorname{det}(A+B) \geqslant \operatorname{det}(A)+\operatorname{det}(B)$.

Combining Lemma 3.7 with equation (3.6), we deduce

$$
\operatorname{det}\left(\sum_{i} x_{i} A_{i}^{\prime}\right) \geqslant \operatorname{det}\left(\sum_{i} x_{i} \in J_{i}\right)=\epsilon^{r} \prod_{j=1}^{r} \sum_{i: r_{i} \geqslant j} x_{i}>0
$$

as required. The second intermediate result is as follows:

CLAIM 3.7.1. There exists a constant $c_{2}>0$ such that for all $1 \leqslant \alpha, \beta \leqslant r$ and all $\left(x_{1}, \ldots, x_{k}\right) \in \mathbb{R}_{>0} \times \mathbb{R}_{\geqslant 0}^{k-1}$ we have the following bound on the cofactors of the matrix $\sum_{i=1}^{k} x_{i} A_{i}^{\prime}$ :

$$
\left|\operatorname{cof}_{\alpha, \beta}\left(\sum_{i=1}^{k} x_{i} A_{i}^{\prime}\right)\right| \leqslant c_{2} \prod_{\substack{\alpha^{\prime}=1 \\ \alpha^{\prime} \neq \min (\alpha, \beta)}}^{r} \sum_{\substack{i: r_{i} \geqslant \alpha^{\prime} \\ x_{i}}} x_{i}
$$

To prove this second claim, write $A=\sum_{i} x_{i} A_{i}^{\prime}$. Then there is a constant $c_{3}$ such that for $1 \leqslant \alpha^{\prime}, \beta^{\prime} \leqslant r$ one has

$$
\left|A_{\alpha^{\prime}, \beta^{\prime}}\right| \leqslant c_{3} \sum_{i: r_{i} \geqslant \max \left(\alpha^{\prime}, \beta^{\prime}\right)} x_{i} \leqslant c_{3} \sum_{i: r_{i} \geqslant \alpha^{\prime}} x_{i} .
$$

Let $\sigma:\{1, \ldots, \hat{\alpha}, \ldots, r\} \stackrel{\sim}{\rightarrow}\{1, \ldots, \hat{\beta}, \ldots, r\}$ be a bijection (the^ means 'omit'). Then

$$
\prod_{\alpha^{\prime} \neq \alpha}\left|A_{\alpha^{\prime}, \sigma\left(\alpha^{\prime}\right)}\right| \leqslant c_{3}^{r-1} \prod_{\alpha^{\prime} \neq \alpha} \sum_{i: r_{i} \geqslant \alpha^{\prime}} x_{i}
$$


and since $A_{\alpha^{\prime}, \sigma\left(\alpha^{\prime}\right)}=A_{\sigma\left(\alpha^{\prime}\right), \alpha^{\prime}}$ we also have

$$
\prod_{\alpha^{\prime} \neq \alpha}\left|A_{\alpha^{\prime}, \sigma\left(\alpha^{\prime}\right)}\right| \leqslant c_{3}^{r-1} \prod_{\alpha^{\prime} \neq \beta} \sum_{i: r_{i} \geqslant \alpha^{\prime}} x_{i} .
$$

Choosing the smaller upper bound of the two we find

$$
\prod_{\alpha^{\prime} \neq \alpha}\left|A_{\alpha^{\prime}, \sigma\left(\alpha^{\prime}\right)}\right| \leqslant c_{3}^{r-1} \prod_{\alpha^{\prime} \neq \min (\alpha, \beta)} \sum_{i: r_{i} \geqslant \alpha^{\prime}} x_{i}
$$

and hence

$$
\left|\operatorname{cof}_{\alpha, \beta}(A)\right| \leqslant(r-1) ! c_{3}^{r-1} \prod_{\alpha^{\prime} \neq \min (\alpha, \beta)} \sum_{i: r_{i} \geqslant \alpha^{\prime}} x_{i} .
$$

This proves the second claim and, consequently, Lemma 3.6.

Proof of Theorem 3.2(2). From Lemma 3.6 we deduce the existence of a constant $c_{4}>0$ such that, for all $1 \leqslant \alpha, \beta \leqslant r$,

$$
\begin{aligned}
\left|\left(\sum x_{i} A_{i}^{\prime} c_{i}^{\prime}\right)_{\alpha}^{t}\left(\sum x_{i} A_{i}^{\prime}\right)_{\alpha, \beta}^{-1}\left(\sum x_{i} A_{i}^{\prime} c_{i}^{\prime}\right)_{\beta}\right| & \leqslant c_{4} \cdot \frac{\left(\sum_{j: r_{j} \geqslant \alpha} x_{j}\right)\left(\sum_{i: r_{i} \geqslant \beta} x_{i}\right)}{\sum_{j: r_{j} \geqslant \min (\alpha, \beta)} x_{j}} \\
& =c_{4} \cdot \sum_{j: r_{j} \geqslant \max (\alpha, \beta)} x_{j}
\end{aligned}
$$

and hence

$$
0 \leqslant f=\sum_{\alpha, \beta}\left(\sum x_{i} A_{i}^{\prime} c_{i}^{\prime}\right)_{\alpha}^{t}\left(\sum x_{i} A_{i}^{\prime}\right)_{\alpha, \beta}^{-1}\left(\sum x_{i} A_{i}^{\prime} c_{i}^{\prime}\right)_{\beta} \leqslant c_{4} \sum_{\alpha, \beta} \sum_{j: r_{j} \geqslant \max (\alpha, \beta)} x_{j} .
$$

This is clearly bounded on $H$. This proves Theorem 3.2(2).

Proof of Theorem 3.2(1). We start by noting that

$$
P=\left(\begin{array}{c|c}
\sum x_{i} A_{i}^{\prime}+B_{11} & B_{12} \\
\hline B_{21} & B_{22}
\end{array}\right),
$$

with $B_{22}$ invertible. Moreover, as $P$ is invertible, so is the Schur complement $\sum x_{i} A_{i}^{\prime}+B_{11}-B_{12} B_{22}^{-1} B_{21}$ of $B_{22}$ in $P$. If we put

$$
Q=\left(\sum x_{i} A_{i}^{\prime}+B_{11}-B_{12} B_{22}^{-1} B_{21}\right)^{-1}
$$

then we get

$$
P^{-1}=\left(\begin{array}{c|c}
Q & -Q B_{12} B_{22}^{-1} \\
\hline-B_{22}^{-1} B_{21} Q & B_{22}^{-1}+B_{22}^{-1} B_{21} Q B_{12} B_{22}^{-1}
\end{array}\right) .
$$


Write $A=\sum x_{i} A_{i}^{\prime}$ and $M=B_{11}-B_{12} B_{22}^{-1} B_{21}$ so that $Q=(A+M)^{-1}$. Recall that $A$ is invertible, so that $Q=\left(\operatorname{Id}_{r}+A^{-1} M\right)^{-1} A^{-1}$.

CLAIM 3.8. There exists $a \kappa^{\prime}>\kappa$ such that the series

$$
A^{-1}-A^{-1} M A^{-1}+A^{-1} M A^{-1} M A^{-1}+\cdots+(-1)^{m}\left(A^{-1} M\right)^{m} A^{-1}+\cdots
$$

converges to $Q$ on $\mathbb{R}_{>\kappa^{\prime}} \times \mathbb{R}_{\geqslant 0}^{k-1} \times K$.

Proof. The entries of the matrix $M$ are continuous functions on the compact set $K$, hence bounded. Let $c$ be the constant of Lemma 3.6, choose

$$
\kappa^{\prime}>\max \left(c r^{2} \max \left(M_{\alpha \beta}\right), c, \kappa\right)
$$

and put $\epsilon=c r^{2} \max \left(M_{\alpha \beta}\right) / \kappa^{\prime}$. Note that $0<\epsilon<1$. Moreover, by Lemma 3.6 and the condition $x_{1} \geqslant \kappa^{\prime}$,

$$
\left(\left|\left(A^{-1} M\right)^{m} A^{-1}\right|\right)_{\alpha \beta} \leqslant \frac{c}{x_{1}} \frac{\left(c \max \left(M_{\alpha \beta}\right) r^{2}\right)^{m}}{\kappa^{\prime m}}<\epsilon^{m} .
$$

It follows that the series converges absolutely. By construction, the limit of the series is $(A+M)^{-1}=Q$ finishing the proof of the claim.

Write $M_{1}=\left(\operatorname{Id}_{r}+M A^{-1}\right)^{-1}$ and $M_{2}=\left(\operatorname{Id}_{r}+A^{-1} M\right)^{-1}$. Then $Q=A^{-1} M_{1}=$ $M_{2} A^{-1}$. An argument similar to that of Claim 3.8 shows that the entries of $M_{1}$ and $M_{2}$ are bounded on the set $\mathbb{R}_{>\kappa^{\prime}} \times \mathbb{R}_{\geqslant 0}^{k-1} \times K$.

We deduce from Lemma 3.6 that there is a constant $c_{2}$ such that

$$
\left|Q_{\alpha \beta}\right| \leqslant \frac{c_{2}}{\sum_{j: r_{j} \geqslant \min (\alpha, \beta)} x_{j}}
$$

on the same set. It follows that

$$
\left|\left(Q\left(\sum x_{i} A_{i}^{\prime} c_{i}^{\prime}\right)\right)_{\beta}\right| \text { and }\left|\left(\left(\sum x_{i} A_{i}^{\prime} c_{i}^{\prime}\right)^{t} Q\right)_{\alpha}\right|
$$

are bounded on $\mathbb{R}_{>\kappa^{\prime}} \times \mathbb{R}_{\geqslant 0}^{k-1} \times K$. Moreover, since $Q-A^{-1}=A^{-1} M_{3} A^{-1}$ with again $M_{3}$ having bounded entries, we deduce that there is another constant $c_{3}$ such that

$$
\left|\left(Q-A^{-1}\right)_{\alpha \beta}\right| \leqslant \frac{c_{3}}{\left(\sum_{j: r_{j} \geqslant \alpha} x_{j}\right)\left(\sum_{i: r_{i} \geqslant \beta} x_{i}\right)},
$$

and consequently

$$
\left|\left(\sum x_{i} A_{i}^{\prime} c_{i}^{\prime}\right)^{t}\left(Q-A^{-1}\right)\left(\sum x_{i} A_{i}^{\prime} c_{i}^{\prime}\right)\right|
$$


is bounded. Finally, to prove that $|\varphi-f|$ is bounded we compute

$$
\begin{aligned}
\varphi-f= & \left(\sum x_{i} A_{i}^{\prime} c_{i}^{\prime}\right)^{t}\left(Q-A^{-1}\right)\left(\sum x_{i} A_{i}^{\prime} c_{i}^{\prime}\right)+2 a_{1}^{t} Q\left(\sum x_{i} A_{i}^{\prime} c_{i}^{\prime}\right) \\
& +a_{1}^{t} Q a_{1}-2 a_{2}^{t} B_{22}^{-1} B_{21} Q\left(\sum x_{i} A_{i}^{\prime} c_{i}^{\prime}\right) \\
& -2 a_{2}^{t} B_{22}^{-1} B_{21} Q a_{1}+a_{2}^{t}\left(B_{22}^{-1}+B_{22}^{-1} B_{21} Q B_{12} B_{22}^{-1}\right) a_{2}
\end{aligned}
$$

and we use the previously obtained bounds. This proves Theorem 3.2(1).

Proof of Theorem 3.2(3). From now on we assume that $k=1$ so we have $\varphi: \mathbb{R}_{>\kappa} \times K \rightarrow \mathbb{R}$ and $f: \mathbb{R}_{>0} \rightarrow \mathbb{R}$. Explicitly,

$$
\varphi\left(x_{1}, \lambda\right)=\left(A_{1} x_{1} c_{1}+a\right)^{t} P^{-1}\left(A_{1} x_{1} c_{1}+a\right)
$$

with $P=A_{1} x_{1}+B$, and $f=c_{1}^{t} A_{1} c_{1} x_{1}$. Recall that we write $\varphi_{0}=\varphi-f$. Put $w_{0}=a-B c_{1}$.

LEMMA 3.9. We have

$$
\varphi_{0}\left(x_{1}, \lambda\right)=2 a^{t} c_{1}-c_{1}^{t} B c_{1}+w_{0}^{t} P^{-1} w_{0} .
$$

Proof. We compute

$$
\begin{aligned}
\varphi_{0}\left(x_{1}, \lambda\right) & =\left(A_{1} x_{1} c_{1}+a\right)^{t} P^{-1}\left(A_{1} x_{1} c_{1}+a\right)-c_{1}^{t} A_{1} c_{1} x_{1} \\
& =\left(w_{0}+P c_{1}\right)^{t} P^{-1}\left(w_{0}+P c_{1}\right)-c_{1}^{t} A_{1} c_{1} x_{1} \\
& =w_{0}^{t} P^{-1} w_{0}+2 c_{1}^{t} w_{0}+c_{1}^{t} P c_{1}-c_{1}^{t} A_{1} c_{1} x_{1} \\
& =w_{0}^{t} P^{-1} w_{0}+2 c_{1}^{t} a-c_{1}^{t} B c_{1} .
\end{aligned}
$$

We continue to assume that $k=1$. It follows that $A_{1}^{\prime}$ is invertible.

LEMMA 3.10. In the above notation and with $k=1$, we have

$$
P^{-1}=\left(\begin{array}{c|c}
0 & 0 \\
\hline 0 & B_{22}^{-1}
\end{array}\right)+\frac{1}{x_{1}}\left(\begin{array}{c|c}
A_{1}^{\prime-1} & -A_{1}^{-1} B_{12} B_{22}^{-1} \\
\hline-B_{22}^{-1} B_{21} A_{1}^{\prime-1} & B_{22}^{-1} B_{21} A_{1}^{\prime-1} B_{12} B_{22}^{-1}
\end{array}\right)+O\left(x_{1}^{-2}\right)
$$

and

$$
P^{-1} A_{1}=\frac{1}{x_{1}}\left(\begin{array}{c|c}
1 & 0 \\
\hline-B_{22}^{-1} B_{21} & 0
\end{array}\right)+O\left(x_{1}^{-2}\right)
$$

as $x_{1} \rightarrow \infty$, where the implicit constants are uniform in $K$. 
Proof. From equation (3.7) we obtain

$$
P^{-1}=\left(\begin{array}{c|c}
0 & 0 \\
\hline 0 & B_{22}^{-1}
\end{array}\right)+\left(\begin{array}{c|c}
Q & -Q B_{12} B_{22}^{-1} \\
\hline-B_{22}^{-1} B_{21} Q & B_{22}^{-1} B_{21} Q B_{12} B_{22}^{-1}
\end{array}\right) .
$$

Also recall that $Q=\left(\operatorname{Id}_{r}+A^{-1} M\right)^{-1} A^{-1}$ with $A=A_{1}^{\prime} x_{1}$ and $M$ bounded. This yields $Q=x_{1}^{-1} A_{1}^{-1}+O\left(x_{1}^{-2}\right)$ as $x_{1} \rightarrow \infty$. The first estimate readily follows. Upon recalling that

$$
A_{1}=\left(\begin{array}{c|c}
A_{1}^{\prime} & 0 \\
\hline 0 & 0
\end{array}\right)
$$

the second estimate also follows.

To finish the proof of Theorem 3.2(3), note that by combining Lemma 3.9 and Lemma 3.10 that

$$
\varphi_{0}\left(x_{1}, \lambda\right)=2 a^{t} c_{1}-c_{1}^{t} B c_{1}+w_{0}^{t}\left(\begin{array}{c|c}
0 & 0 \\
\hline 0 & B_{22}^{-1}
\end{array}\right) w_{0}+O\left(x_{1}^{-1}\right)
$$

as $x_{1} \rightarrow \infty$. From this it is immediate that $\varphi_{0}$ extends continuously to a function from $\overline{\mathbb{R}_{>\kappa}} \times K$ to $\mathbb{R}$. Next, from Lemma 3.9 we have

$$
\frac{\partial \varphi_{0}}{\partial x_{1}}=-w_{0}^{t} P^{-1} A_{1} P^{-1} w_{0}, \quad \frac{\partial^{2} \varphi_{0}}{\partial x_{1}^{2}}=2 w_{0}^{t} P^{-1} A_{1} P^{-1} A_{1} P^{-1} w_{0} .
$$

Combining this with Lemma 3.10 we find the estimates

$$
\frac{\partial \varphi_{0}}{\partial x_{1}}=O\left(x_{1}^{-2}\right), \quad \frac{\partial^{2} \varphi_{0}}{\partial x_{1}^{2}}=O\left(x_{1}^{-3}\right),
$$

completing the proof of Theorem 3.2(3).

3.4. On the recession function of a normlike function. Let $f: \mathbb{R}_{>0}^{k} \rightarrow \mathbb{R}$ be the recession function of the normlike function $\varphi$ associated to $\left(\left(A_{i}\right),\left(c_{i}\right), a, B\right)$ as above. The purpose of this section is to list a number of useful properties of $f$.

PROPOSITION 3.11. The function $f$ is convex, that is, for all $x, y \in \mathbb{R}_{>0}^{k}$ and all $\lambda \in[0,1]$ we have $f(\lambda x+(1-\lambda) y) \leqslant \lambda f(x)+(1-\lambda) f(y)$.

Proof. Example 3.4 on [5, page 90] states that the function $h_{g}: \mathbb{R}^{g} \times S_{g}^{++}(\mathbb{R}) \rightarrow \mathbb{R}$ given by $h_{g}(x, Y)=x^{t} Y^{-1} x$ is convex. The function $f: \mathbb{R}_{>0}^{k} \rightarrow \mathbb{R}$ is the composition of $h_{g}$ with the linear map

$$
\mathbb{R}_{>0}^{k} \rightarrow \mathbb{R}^{g} \times S_{g}^{++}(\mathbb{R}), \quad\left(x_{1}, \ldots, x_{k}\right) \mapsto\left(\sum_{i=1}^{k} x_{i} A_{i}^{\prime} c_{i}^{\prime}, \sum_{i=1}^{k} x_{i} A_{i}^{\prime}\right) .
$$


Since the composition of a linear map followed by a convex function is again convex, we deduce that $f$ is convex.

Proposition 3.12. The function $f$ extends to a continuous function $\bar{f}: \mathbb{R}_{\geqslant 0}^{k} \rightarrow$ $\mathbb{R}_{\geqslant 0}$. The function $\bar{f}$ is homogeneous of weight one and convex.

Proof. By Theorem 3.2(2) we know that the function $f$ is bounded on the open standard simplex $\Delta^{0}$. Define

$$
\overline{\bar{f}}: \Delta \rightarrow \mathbb{R}_{\geqslant 0}
$$

by the formula

$$
\overline{\bar{f}}\left(x_{1}, \ldots, x_{k}\right)=\inf _{\left(p_{l}\right)_{l} \rightarrow\left(x_{1}, \ldots, x_{k}\right)} \liminf _{l \rightarrow \infty} f\left(p_{l}\right) ;
$$

here the infimum is over sequences in $\Delta^{0}$ tending to the point $\left(x_{1}, \ldots, x_{k}\right)$. This function $\overline{\bar{f}}$ is well defined because $f$ is bounded on $\Delta^{0}$. It follows easily from the definition of $\overline{\bar{f}}$ that $\overline{\bar{f}}$ is convex and lower semicontinuous. Since $\Delta$ is a convex polytope, it follows from [23, Theorem 10.2] that $\overline{\bar{f}}$ is continuous. Now extend $\overline{\bar{f}}$ to $\mathbb{R}_{\geqslant 0}^{k} \backslash\{0\}$ by homogeneity. By sending in addition 0 to 0 we obtain the required continuous and convex function $\bar{f}: \mathbb{R}_{\geqslant 0}^{k} \rightarrow \mathbb{R}_{\geqslant 0}$.

We can make the function $\bar{f}$ explicit as follows. Let $I \subseteq\{1, \ldots, k\}$ be any subset, and set $J=\{1, \ldots, k\} \backslash I$. We consider the restriction of $\bar{f}$ to the subset $\mathbb{R}_{>0}^{I} \subseteq \mathbb{R}_{\geqslant 0}^{k}$ given by setting $x_{j}$ equal to zero for all $j \in J$. Let

$$
r_{I}=\operatorname{rk}\left(\sum_{i \in I} x_{i} A_{i}\right): x_{i}>0,
$$

and for $i \in I$ set

$$
A_{i}=\left(\begin{array}{c|c|c}
A_{i}^{\prime \prime} & 0 \\
\hline 0 & 0
\end{array}\right)
$$

where $A_{i}^{\prime \prime}$ has size $r_{I}$, and similarly

$$
c_{i}=\left(\frac{c_{i}^{\prime \prime}}{\star}\right),
$$

where $c_{i}^{\prime \prime}$ has length $r_{I}$.

Note that, if $I \neq \emptyset$, then $r_{I} \geqslant 1$. Let $K \subset \mathbb{R}_{>0}^{J}$ be an arbitrary compact subset. Write $x_{I}=\left(x_{i}\right)_{i \in I}$ and $x_{J}=\left(x_{j}\right)_{j \in J}$. We define the function

$$
f_{I}: \mathbb{R}_{>0}^{I} \times K \rightarrow \mathbb{R}, \quad\left(x_{I} ; x_{J}\right) \mapsto f\left(x_{1}, \ldots, x_{k}\right) .
$$


Write

$$
a\left(x_{J}\right)=\sum_{j \in J} x_{j} A_{j}^{\prime} c_{j}^{\prime}, \quad B\left(x_{J}\right)=\sum_{j \in J} A_{j}^{\prime} x_{j},
$$

then we see that

$$
f_{I}\left(x_{I} ; x_{J}\right)=\left(\sum_{i \in I} x_{i} A_{i}^{\prime} c_{i}^{\prime}+a\left(x_{J}\right)\right)^{t}\left(\sum_{i \in I} x_{i} A_{i}^{\prime}+B\left(x_{J}\right)\right)^{-1}\left(\sum_{i \in I} x_{i} A_{i}^{\prime} c_{i}^{\prime}+a\left(x_{J}\right)\right)
$$

and hence by Theorem $3.2 f_{I}$ has a recession function $\operatorname{rec}\left(f_{I}\right): \mathbb{R}_{>0}^{I} \rightarrow \mathbb{R}$ which can be written as

$$
\operatorname{rec}\left(f_{I}\left(x_{I}\right)\right)=\left(\sum_{i \in I} x_{i} A_{i}^{\prime \prime} c_{i}^{\prime \prime}\right)^{t}\left(\sum_{i \in I} x_{i} A_{i}^{\prime \prime}\right)^{-1}\left(\sum_{i \in I} x_{i} A_{i}^{\prime \prime} c_{i}^{\prime \prime}\right),
$$

when $I \neq \emptyset$, and $\operatorname{rec}\left(f_{\emptyset}\right)=0$. Note that $\operatorname{rec}\left(f_{I}\right)$ is independent of the choice of $K$. Also note that, by Theorem $3.2,\left|f_{I}-\operatorname{rec}\left(f_{I}\right)\right|$ is bounded on $\mathbb{R}_{>0}^{I} \times K$.

Proposition 3.13. Let $I \subseteq\{1, \ldots, k\}$ be any subset. We have

$$
\left.\bar{f}\right|_{\mathbb{R}_{>0}^{I}}=\operatorname{rec}\left(f_{I}\right)
$$

Proof. When $I=\emptyset$ the equality is trivially true. We assume that $I \neq \emptyset$. Choose $c \in \mathbb{R}_{>0}^{J}$ and $x_{I} \in \mathbb{R}_{>0}^{I}$ arbitrarily. By Theorem 3.2(1) with $K=\{c\}$, there exists a constant $\delta>0$ depending on $c$ and $x_{I}$ such that for all $\lambda \in \mathbb{R}_{>0}$ we have

$$
\left|\left(\operatorname{rec}\left(f_{I}\right)\right)\left(\lambda x_{I}\right)-f_{I}\left(\lambda x_{I} ; c\right)\right| \leqslant \delta .
$$

We deduce that for all $\lambda \in \mathbb{R}_{>0}$ we have

$$
\left|\left(\operatorname{rec}\left(f_{I}\right)\right)\left(x_{I}\right)-f\left(x_{I}, \frac{c}{\lambda}\right)\right| \leqslant \frac{\delta}{\lambda} .
$$

As $\bar{f}$ extends $f$ continuously we have

$$
\lim _{\lambda \rightarrow \infty} f\left(x_{I}, \frac{c}{\lambda}\right)=\left.\bar{f}\right|_{\mathbb{R}_{>0}^{I}}\left(x_{I}\right)
$$

independently of the choice of $c$. Combining with the bound (3.9), we find that

$$
\left(\operatorname{rec}\left(f_{I}\right)\right)\left(x_{I}\right)=\left.\bar{f}\right|_{\mathbb{R}_{>0}^{I}}\left(x_{I}\right),
$$

as required. 
A special case of interest is when $|I|=1$. For each $1 \leqslant i \leqslant k$, set

$$
A_{i}=\left(\begin{array}{c|c}
A_{i}^{\mathrm{e}} & 0 \\
\hline 0 & 0
\end{array}\right),
$$

where $A_{i}^{\mathrm{e}}$ has size $r_{i}=\operatorname{rk} A_{i}$ and hence is positive definite; here e is short for 'essential'. Similarly set

$$
c_{i}=\left(\frac{c_{i}^{\mathrm{e}}}{\star}\right),
$$

where $c_{i}^{\mathrm{e}}$ has length $r_{i}$. Define

$$
\mu_{i}=c_{i}^{t} A_{i} c_{i}=\left(c_{i}^{\mathrm{e}}\right)^{t} A_{i}^{\mathrm{e}} c_{i}^{\mathrm{e}} .
$$

Then $\mu_{i} \geqslant 0$ and we have for all $x_{i}>0$

$\bar{f}\left(0, \ldots, 0, x_{i}, 0, \ldots, 0\right)=\left(\operatorname{rec}\left(f_{\{i\}}\right)\right)\left(x_{i}\right)=x_{i}\left(c_{i}^{\mathrm{e}}\right)^{t}\left(A_{i}^{\mathrm{e}}\right)^{t} x_{i}^{-1}\left(A_{i}^{\mathrm{e}}\right)^{-1} x_{i} A_{i}^{\mathrm{e}} c_{i}^{\mathrm{e}}=x_{i} \mu_{i}$.

In particular, the function $\bar{f}\left(0, \ldots, 0, x_{i}, 0, \ldots, 0\right)$ is homogeneous linear in $x_{i}$, and

$$
\mu_{i}=\bar{f}(0, \ldots, 0,1,0, \ldots, 0) .
$$

We call $\mu_{1}, \ldots, \mu_{k} \geqslant 0$ the coefficients associated to $\varphi$.

\section{Proofs of the main results}

In this section we prove our main results. We will continue to work with the 'diagonal case' where we consider the pullback Poincaré bundle $\mathcal{P}_{v}$ associated to a single admissible normal function $v$ of our family $\pi: Y \rightarrow X$. As was explained at the beginning of Section 2.3, by the biextension property of the Poincare bundle this is sufficient for the purpose of proving the main results as stated in the introduction.

4.1. Singularities of the biextension metric. In this section we will prove Theorem 1.1.

Proof of Theorem 1.1. Following Theorem 2.14, take

- a small enough $\epsilon>0$,

- matrices

$$
A_{1}, \ldots, A_{k} \in S_{g}(\mathbb{R})
$$

of positive rank such that $\Delta^{-1} A_{i} \in M_{g}(\mathbb{Z})$ for $i=1, \ldots, k$, 
- vectors

$$
c_{1}, \ldots, c_{k} \in \mathbb{Q}^{g}
$$

such that $\Delta^{-1} A_{i} c_{i} \in \mathbb{Z}^{g}$ for $i=1, \ldots, k$,

- bounded holomorphic maps $\alpha: \Delta_{\epsilon}^{n} \rightarrow \mathbb{C}^{g}$ and $\psi: \Delta_{\epsilon}^{n} \rightarrow \mathbb{P}^{g}$,

such that the multivalued period mapping

$$
(\Omega, \delta): U_{\epsilon} \cap X \rightarrow \mathcal{M}=\mathbb{H}_{g} \times \mathbb{C}^{g}
$$

of the variation of mixed Hodge structures $\boldsymbol{H}(v)$ on $U_{\epsilon}$ is given by the formula

$$
\left.(\underline{q})=\left(q_{1}, \ldots, q_{n}\right) \mapsto\left(\sum_{j=1}^{k} A_{j} \frac{\log q_{j}}{2 \pi i}+\psi(\underline{q}), \sum_{j=1}^{k} A_{j} c_{j} \frac{\log q_{j}}{2 \pi i}+\alpha \underline{q}\right)\right)
$$

(recall that $U_{\epsilon}$ was defined in Section 1.3). Put $a=2 \pi \operatorname{Im} \alpha, B=2 \pi \operatorname{Im} \psi$, and define $\kappa \in \mathbb{R}$ via $\kappa=-\log \epsilon$. As above define the function $\varphi: \mathbb{R}_{>\kappa}^{k} \times \Delta_{\epsilon}^{n} \rightarrow \mathbb{R}_{\geqslant 0}$ via

$$
\varphi\left(x_{1}, \ldots, x_{k} ; \underline{q}\right)=\left(\sum_{i=1}^{k} x_{i} A_{i} c_{i}+a\right)^{t}\left(\sum_{i=1}^{k} x_{i} A_{i}+B\right)^{-1}\left(\sum_{i=1}^{k} x_{i} A_{i} c_{i}+a\right) .
$$

Choose any $0<\epsilon^{\prime}<\epsilon$. The restriction of $\varphi$ to $\mathbb{R}_{>\kappa}^{k} \times \bar{\Delta}_{\epsilon^{\prime}}^{n}$ is then a normlike function of dimension $k$. Let $f: \mathbb{R}_{>0}^{k} \rightarrow \mathbb{R}_{\geqslant 0}$ be the associated recession function $f=\operatorname{rec}(\varphi)$. Recalling the explicit expression (3.3) for $f$, the conditions

$$
\Delta^{-1} A_{i} \in S_{g}(\mathbb{R}) \cap M_{g}(\mathbb{Z}), \quad \Delta^{-1} A_{i} c_{i} \in \mathbb{Z}^{g},
$$

for each $i=1, \ldots, k$ imply that the $A_{i}$ and $A_{i} c_{i}$ are themselves integral and that $f$ is the quotient of two homogeneous polynomials in $\mathbb{Z}\left[x_{1}, \ldots, x_{k}\right]$. In particular, $f \in \mathbb{Q}\left(x_{1}, \ldots, x_{k}\right)$. It is clear that $f$ is homogeneous of weight one, and by Proposition 3.11 the function $f$ is convex when seen as a real-valued function on $\mathbb{R}_{>0}^{k}$.

Let $s$ be an admissible section of $\mathcal{P}_{\nu}$ over $U_{\epsilon} \cap X$. Following Corollary 2.16 we have

$$
-\log \|s\|=-\log |h|+\varphi\left(-\log \left|q_{1}\right|, \ldots,-\log \left|q_{k}\right| ; \underline{q}\right)
$$

on $U_{\epsilon} \cap X$ with $h$ a meromorphic function on $U_{\epsilon}$, holomorphic on $U_{\epsilon} \cap X$. As $s$ is locally generating over $U_{\epsilon} \cap X$ we have that $h$ has no zeros or poles on $U_{\epsilon} \cap X$. Hence there is a linear form $l \in \mathbb{Z}\left[x_{1}, \ldots, x_{k}\right]$ and a holomorphic map $u: U_{\epsilon} \rightarrow \mathbb{C}^{*}$ such that

$$
-\log |h|=l\left(-\log \left|q_{1}\right|, \ldots,-\log \left|q_{k}\right|\right)+\log |u|
$$

on $U_{\epsilon} \cap X$. The image of $\bar{U}_{\epsilon^{\prime}}$ under the map $u$ is compact. 
Put $f_{s}=f+l$ in $\mathbb{Q}\left(x_{1}, \ldots, x_{k}\right)$. Then $f_{s}$ is again homogeneous of weight one and convex as a function on $\mathbb{R}_{>0}^{k}$. Our claim is that $f_{s}$ satisfies all the requirements of Theorem 1.1. We need to show first of all that $-\log \|s\|-f_{s}\left(-\log \left|q_{1}\right|, \ldots\right.$, $\left.-\log \left|q_{k}\right|\right)$ is bounded on $\bar{U}_{\epsilon^{\prime}} \cap X$ and extends continuously over $\bar{U}_{\epsilon^{\prime}} \backslash D^{\text {sing }}$.

In order to see this, put $\varphi_{0}=\varphi-f$ on $\mathbb{R}_{>\kappa}^{k} \times \Delta_{\epsilon}^{n}$. Then

$$
\begin{aligned}
-\log \|s\|(\underline{q})= & f_{s}\left(-\log \left|q_{1}\right|, \ldots,-\log \left|q_{k}\right|\right)+\log |u| \\
& +\varphi_{0}\left(-\log \left|q_{1}\right|, \ldots,-\log \left|q_{k}\right| ; \underline{q}\right)
\end{aligned}
$$

on $U_{\epsilon} \cap X$. Note that $\log |u|$ extends in a continuous and bounded manner over the whole of $\bar{U}_{\epsilon^{\prime}}$. We are reduced to showing that $\varphi_{0}\left(-\log \left|q_{1}\right|, \ldots,-\log \left|q_{k}\right| ; \underline{q}\right)$ is bounded on $\bar{U}_{\epsilon^{\prime}} \cap X$ and extends continuously over $\bar{U}_{\epsilon^{\prime}} \backslash D^{\text {sing }}$.

For this we invoke Theorem 3.2(1). This readily gives the boundedness of $\varphi_{0}$ via the map

$$
(-\log |\cdot|, \mathrm{id}):\left(\Delta_{\epsilon}^{*}\right)^{k} \times \Delta_{\epsilon}^{n} \rightarrow \mathbb{R}_{>\kappa}^{k} \times \Delta_{\epsilon}^{n} .
$$

Let $p \in\left(D \backslash D^{\text {sing }}\right) \cap \bar{U}_{\epsilon^{\prime}}$. Up to a change in the order of the variables, we can assume that the coordinates of $p$ satisfy $q_{1}=0, q_{i} \neq 0$ for $i=2, \ldots, N$. We take a small polydisk $V_{\epsilon^{\prime \prime}} \subset \bar{U}_{\epsilon^{\prime}}$ of small radius $\epsilon^{\prime \prime}$ with center at $p$ such that $V_{\epsilon^{\prime \prime}} \cap X$ can be identified with $\Delta_{\epsilon^{\prime \prime}}^{*} \times \Delta_{\epsilon^{\prime \prime}}^{n-1}$ and hence $V_{\epsilon^{\prime \prime}} \cap D$ can be identified with the divisor $q_{1}=0$ on $\Delta_{\epsilon^{\prime \prime}}^{n}$. Write

$$
\underline{r}=\left(r_{2}, \ldots, r_{k}\right)=\left(-\log \left|q_{2}\right|, \ldots,-\log \left|q_{k}\right|\right)
$$

for $\underline{q} \in V_{\epsilon^{\prime \prime}}$; then $\underline{r}$ can be assumed to move through a compact subset $K^{\prime} \subset \mathbb{R}^{k-1}$. Put $K^{\prime \prime}=K^{\prime} \times \bar{\Delta}_{\epsilon^{\prime}}^{n}$. We define functions $\varphi^{\prime}: \mathbb{R}_{>\kappa} \times K^{\prime \prime} \rightarrow \mathbb{R}_{\geqslant 0}$ and $f^{\prime}: \mathbb{R}_{>\kappa} \times$ $K^{\prime \prime} \rightarrow \mathbb{R}_{\geqslant 0}$ via

$$
\varphi^{\prime}\left(x_{1} ; \underline{r}, \underline{q}\right)=\varphi\left(x_{1}, \underline{r} ; \underline{q}\right), \quad f^{\prime}\left(x_{1} ; \underline{r}\right)=f\left(x_{1}, \underline{r}\right) .
$$

Then both $\varphi^{\prime}, f^{\prime}$ are normlike of dimension one. Write

$$
A_{1}=\left(\begin{array}{c|c}
A_{1}^{\prime} & 0 \\
\hline 0 & 0
\end{array}\right), \quad A_{1}^{\prime}=\left(\begin{array}{c|c|c}
A_{1}^{\prime \prime} & 0 \\
\hline 0 & 0
\end{array}\right),
$$

with $A_{1}^{\prime}$ positive semidefinite of size $r$, and $A_{1}^{\prime \prime}=A_{1}^{\mathrm{e}}$ positive definite of size and rank $r_{1}$. Then it is readily verified that $\operatorname{both} \operatorname{rec}\left(f^{\prime}\right)$ and $\operatorname{rec}\left(\varphi^{\prime}\right)$ are equal to the linear function $x_{1} \mu_{1}=x_{1} c_{1}^{t} A_{1} c_{1}=\bar{f}\left(x_{1}, 0, \ldots, 0\right)$. Note that

$$
\varphi_{0}\left(-\log \left|q_{1}\right|, \ldots,-\log \left|q_{k}\right| ; \underline{q}\right)=\varphi^{\prime}\left(-\log \left|q_{1}\right| ; \underline{r}, \underline{q}\right)-f^{\prime}\left(-\log \left|q_{1}\right| ; \underline{r}\right)
$$

on $V_{\epsilon^{\prime}} \cap X$. We are done once we show that $\varphi^{\prime}-f^{\prime}$ extends continuously over $\overline{\mathbb{R}_{>\kappa}} \times K^{\prime \prime}$. Following Theorem 3.2(3) we have that both $\varphi^{\prime}-\operatorname{rec}\left(\varphi^{\prime}\right)$ and $f^{\prime}-$ 
$\operatorname{rec}\left(f^{\prime}\right)$ extend continuously over $\overline{\mathbb{R}_{>\kappa}} \times K^{\prime \prime}$. As $\operatorname{rec}\left(\varphi^{\prime}\right)=\operatorname{rec}\left(f^{\prime}\right)$ we find the required extension result.

The second item of Theorem 1.1 is clear. As $f_{s}$ is up to a linear form the recession function of a normlike function we have that $f_{s}$ is convex, and by Proposition 3.12 that $f_{s}$ extends as a convex, continuous homogeneous weight-one function $\bar{f}_{s}: \mathbb{R}_{\geqslant 0}^{k} \rightarrow \mathbb{R}$. This finally proves items (3) and (4) of Theorem 1.1.

4.2. The Lear extension made explicit. Let $s$ be an admissible section of $\mathcal{P}=\mathcal{P}_{\nu}$. We recall that this means that $s$ corresponds to an admissible biextension variation of mixed Hodge structures on $X$. Then $s$ can also be seen as a rational section of the Lear extension $[\mathcal{P},\|-\|]_{\bar{X}}$ of $\mathcal{P}$ over $\bar{X}$. We can now compute the global $\mathbb{Q}$-divisor $\operatorname{div}_{\bar{X}}(s)$ that represents $[\mathcal{P},\|-\|]_{\bar{X}}$. We do this after a little digression.

Write $U=U_{\epsilon^{\prime}}, V=V_{\epsilon^{\prime \prime}}$ to reduce notation. Let $\mu_{1}, \ldots, \mu_{k} \in \mathbb{Q}$ be the coefficients of $\varphi$ (see end of Section 3.4 for the definition), and $v_{i}=\operatorname{ord}_{D_{i}} h$ for $i=1, \ldots, k$, and $a_{i}=\mu_{i}+v_{i}$. Here $D_{i}$ is the divisor on $\bar{X}$ given locally on $U$ by $q_{i}=0$. We obtain from the above proof that

$$
-\log \|s\|(\underline{q})=-a_{1} \log \left|q_{1}\right|+\psi_{1}(\underline{q})
$$

on $V \cap X$ where $\psi_{1}(\underline{q})$ extends continuously over $V$.

We say that $p \in \bar{X}$ is of depth $k$ if $p$ is on precisely $k$ of the irreducible divisors $D_{i}$. The set $\Sigma_{k}$ of points of depth $k$ on $\bar{X}$ is a locally closed subset of $\bar{X}$ and for $k \geqslant 1$ they yield a stratification of $D=\bar{X} \backslash X$. For $p \in \Sigma_{k}$ take a coordinate neighborhood $U \subset \bar{X}$ such that $p=(0, \ldots, 0)$ and $D \cap X$ is given by the equation $q_{1} \cdots q_{k}=0$. Theorem 1.1 yields an associated homogeneous weightone function $f_{p, s} \in \mathbb{Q}\left(x_{1}, \ldots, x_{k}\right)$.

Lemma 4.1. The map $\Sigma_{k} \rightarrow \mathbb{Q}\left(x_{1}, \ldots, x_{k}\right)$ given by $p \mapsto f_{p, s}$ is locally constant.

Proof. Take $p, U$ as above and let $y=\left(0, \ldots, 0, y_{k+1}, \ldots, y_{n}\right) \in U$ be another point of depth $k$. Let $q_{i}^{\prime}=q_{i}$ for $i=1, \ldots, k, q_{i}^{\prime}=q_{i}-y_{i}$ for $i=k+1, \ldots, n$. Then $\underline{q}^{\prime}$ are coordinates centered around $y$ and we have

$$
\begin{aligned}
-\log \|s\| & =f_{p, s}\left(-\log \left|q_{1}\right|, \ldots,-\log \left|q_{k}\right|\right)+\psi_{p}(\underline{q}) \\
& =f_{y, s}\left(-\log \left|q_{1}^{\prime}\right|, \ldots,-\log \left|q_{k}^{\prime}\right|\right)+\psi_{y}\left(\underline{q^{\prime}}\right) \\
& =f_{y, s}\left(-\log \left|q_{1}\right|, \ldots,-\log \left|q_{k}\right|\right)+\psi_{y}\left(q_{i}-y_{i}\right)
\end{aligned}
$$


on $U \cap X$ with $\psi_{p}, \psi_{y}$ bounded on $U \cap X$. We find that $f_{p, s}-f_{y, s}$ is bounded on $\mathbb{R}_{>\kappa}^{k}$ and, being homogeneous of weight one, it vanishes identically.

In order to compute the $\operatorname{divisor}_{\operatorname{div}_{\bar{X}}}(s)$ that represents the Lear extension of $\mathcal{P}_{v}$ over $\bar{X}$ we are interested in the behavior of the function $f_{s}: \Sigma_{1} \rightarrow \mathbb{Q}(x)$ obtained from Lemma 4.1 by restricting to $k=1$. Note that $\Sigma_{1}=D \backslash D^{\text {sing }}$. Let $D=\bigcup_{\alpha=1}^{d} D_{\alpha}$ be the decomposition of $D$ into irreducible components. Take any irreducible component $D_{\alpha}$. Since $D_{\alpha} \backslash D^{\text {sing }}$ is connected, we deduce from Lemma 4.1 that the function

$$
f_{s, \alpha}: D_{\alpha} \backslash D^{\text {sing }} \rightarrow \mathbb{Q}(x)
$$

is constant. Its value is a homogeneous linear function which we write as $f_{s, \alpha}(x)=a_{\alpha} x$, with $a_{\alpha} \in \mathbb{Q}$. In this notation we find:

COROLlaRY 4.2. Let $s$ be a section of $\mathcal{P}$ corresponding to an admissible biextension variation on $X$. Let $L=[\mathcal{P},\|-\|]_{\bar{X}}$ be the Lear extension of $\mathcal{P}$ over $\bar{X}$. Then $L$ is represented by the $\mathbb{Q}$-divisor

$$
\operatorname{div}_{\bar{X}}(s)=\sum_{\alpha=1}^{d} a_{\alpha} D_{\alpha}
$$

on $\bar{X}$.

4.3. Local integrability. Our next task is to investigate $\partial \bar{\partial} \log \|s\|$ over curves.

Proof of Theorem 1.3. We use the estimates from Theorem 3.2(3). We assume $k=n=1$, but otherwise keep the notation and assumptions from Section 4.1. In particular, we have the normlike function $\varphi\left(x_{1}, q_{1}\right)$ on $\mathbb{R}_{>\kappa} \times \Delta_{\epsilon}$ and the associated recession function $f=\operatorname{rec}(\varphi)$ on $\mathbb{R}_{>0}$. Put $\varphi_{0}=\varphi-f$. Put $\varphi_{1}\left(q_{1}\right)=\varphi_{0}\left(-\log \left|q_{1}\right|\right.$, $\left.q_{1}\right)$. By Corollary 2.16 on $U_{\epsilon} \cap X$, noting that $f$ is linear, we have

$$
-\log \|s\|\left(q_{1}\right)=-\log |h|\left(q_{1}\right)+\varphi_{1}\left(q_{1}\right)
$$

for some meromorphic function $h$. Note that

$$
\partial \varphi_{1}=-\frac{1}{2} \frac{\partial \varphi_{0}}{\partial x_{1}} \frac{d q_{1}}{q_{1}}+\frac{\partial \varphi_{0}}{\partial q_{1}} d q_{1} .
$$

Here $\partial \varphi_{0} / \partial q_{1}$ is smooth and bounded on $\overline{U_{\epsilon^{\prime}}}$, and by Theorem 3.2(3) we have a constant $c_{1}$ such that

$$
\left|\frac{\partial \varphi_{0}}{\partial x_{1}}\right| \leqslant c_{1} \cdot x_{1}^{-2} .
$$


Hence for a smooth vector field $T$ with bounded coefficients we find a constant $c_{2}$ such that

$$
\left|\partial \varphi_{1}(T)\right| \leqslant c_{2} \cdot \frac{1}{\left(-\log \left|q_{1}\right|\right)^{2}\left|q_{1}\right|}
$$

on $U_{\epsilon} \cap X$. A similar argument yields

$$
\left|\bar{\partial} \varphi_{1}(T)\right| \leqslant c_{2} \cdot \frac{1}{\left(-\log \left|q_{1}\right|\right)^{2}\left|q_{1}\right|}
$$

on $U_{\epsilon} \cap X$. In particular, there is a constant $c_{3}$ such that

$$
\left\|\int_{\partial U_{\epsilon}} \partial \varphi_{1}\right\| \leqslant c_{3} \frac{\epsilon}{(\log \epsilon)^{2} \epsilon} .
$$

Thus the residue $\operatorname{res}_{0}\left(\partial \varphi_{1}\right)$ of $\partial \varphi_{1}$ at zero is zero.

Next, there exists a smooth $(1,1)$-form $\zeta$ on $U_{\epsilon}$ such that

$$
\partial \bar{\partial} \varphi_{1}=\frac{1}{4} \frac{\partial^{2} \varphi_{0}}{\partial x_{1}^{2}} \frac{1}{\left|q_{1}\right|^{2}} d q_{1} d \overline{q_{1}}+\zeta .
$$

By Theorem 3.2(3) we have a constant $c_{4}$ such that

$$
\left|\frac{\partial^{2} \varphi_{0}}{\partial x_{1}^{2}}\right| \leqslant c_{4} \cdot x_{1}^{-3} .
$$

Hence for smooth vector fields $T, U$ with bounded coefficients we find a constant $c_{5}$ and an estimate

$$
\left|\partial \bar{\partial} \varphi_{1}(T, U)\right| \leqslant c_{5} \cdot \frac{1}{\left(-\log \left|q_{1}\right|\right)^{3}\left|q_{1}\right|^{2}}
$$

on $U_{\epsilon} \cap X$. This shows that $\partial \bar{\partial} \varphi_{1}$ is locally integrable on $U_{\epsilon}$.

4.4. Effectivity of the height jump divisor. In this section we prove Theorem 1.5. We continue again with the notation as in Section 4.1. In particular, we have $U=U_{\epsilon}$, we have $s$ an admissible section of $\mathcal{P}_{v}$ on $U \cap X$, and $f_{s}: \mathbb{R}_{>0}^{k} \rightarrow \mathbb{R}$ the associated homogeneous weight-one function such that

$$
-\log \|s\|-f_{s}\left(-\log \left|q_{1}\right|, \ldots,-\log \left|q_{k}\right|\right)
$$

is bounded on $U \cap X$ and extends continuously over $\bar{X} \backslash D^{\text {sing }}$. Moreover $f_{s}$ extends as a convex homogeneous weight-one function $\bar{f}_{s}: \mathbb{R}_{\geqslant 0}^{k} \rightarrow \mathbb{R}$ (see 
Theorem 1.1). It is clear that a convex homogeneous weight-one function is subadditive, hence we have the estimate

$$
\bar{f}_{s}\left(x_{1}, \ldots, x_{k}\right) \leqslant \sum_{i=1}^{k} \bar{f}_{s}\left(0, \ldots, 0, x_{i}, 0, \ldots, 0\right)
$$

on $\mathbb{R}_{\geqslant 0}^{k}$.

Now let $\bar{\phi}: \bar{C} \rightarrow \bar{X}$ be a map from a smooth curve, sending a point 0 in $\bar{C}$ to $p=(0, \ldots, 0)$, and such that there exists an open neighborhood $V$ of 0 in $\bar{C}$ such that $\bar{\phi}$ maps $V$ into $U$. We also assume that $\bar{\phi}$ does not map $V$ into $D$. Then $\bar{\phi}$ is given locally at $0 \in \bar{C}$ by

$$
\bar{\phi}(t)=\left(t^{m_{1}} u_{1}, \ldots, t^{m_{i}} u_{i}, \ldots\right),
$$

where $t$ is a local coordinate on $\bar{C}$ at 0 , the $m_{i}$ are nonnegative integers, and $u_{i}$ are units. Write $\phi$ for the restriction of $\bar{\phi}$ to $V \backslash\{0\}$.

Proposition 4.3. We have an equality of $\mathbb{Q}$-divisors on $V$ :

$$
\left.\operatorname{div}\left(\phi^{*} s\right)\right|_{V}=\bar{f}_{s}\left(m_{1}, \ldots, m_{k}\right) \cdot[0],
$$

where $\phi^{*}$ s is viewed as a rational section of the Lear extension $\left[\phi^{*}\left(\mathcal{P}_{\nu},\|-\|\right)\right]_{V}$.

Proof. It suffices to show that

$$
-\log \left\|\phi^{*} s\right\| \sim-\bar{f}_{s}\left(m_{1}, \ldots, m_{k}\right) \log |t|
$$

on $V \backslash\{0\}$, where $\sim$ denotes that the difference is bounded and extends continuously over $V$. As by Theorem 1.1

$$
-\log \|s\|-f_{s}\left(-\log \left|q_{1}\right|, \ldots,-\log \left|q_{k}\right|\right)
$$

is bounded on $U \cap X$ we obtain the boundedness by pullback along $\phi$. The continuous extendability over $V$ then follows from the boundedness combined with the existence of a Lear extension for $\phi^{*}\left(\mathcal{P}_{\nu},\|-\|\right)$.

Proposition 4.4. We have an equality of divisors on $V$ :

$$
\phi^{*}\left(\operatorname{div}_{\bar{X}}(s)\right)=\sum_{i=1}^{k} \bar{f}_{s}\left(0, \ldots, 0, m_{i}, 0, \ldots, 0\right) \cdot[0],
$$

where $s$ is viewed as a rational section of the Lear extension $\left[\mathcal{P}_{v},\|-\|\right]_{U}$. 
Proof. This follows immediately from Corollary 4.2.

Proof of Theorem 1.5. Combining Propositions 4.3 and 4.4 one sees that the line bundle

$$
\left[\phi^{*}\left(\mathcal{P}_{\nu},\|-\|\right)\right]_{\bar{C}}^{\otimes-1} \otimes \bar{\phi}^{*}\left[\mathcal{P}_{\nu},\|-\|\right]_{\bar{X}}
$$

has a canonical nonzero rational section, whose divisor is

$$
\left(-\bar{f}_{s}\left(m_{1}, \ldots, m_{k}\right)+\sum_{i=1}^{k} \bar{f}_{s}\left(0, \ldots, 0, m_{i}, 0, \ldots, 0\right)\right) \cdot[0]
$$

on $V$, which is indeed independent of the choice of rational section $s$. This divisor is effective by the subadditivity of $f_{s}$ expressed by inequality (4.4). In particular, the section is global.

\section{Acknowledgements}

We would like to thank R. Hain and G. Pearlstein for several discussions and useful hints. We also are very grateful to the referees for a number of important corrections and clarifications. Finally we would like to thank the hospitality of the Mathematical Institute of Leiden University and the Instituto de Ciencias Matemáticas where the authors could meet to work on this paper. The first author has been partially supported by the MINECO research projects MTM2013-42135$\mathrm{P}$ and MTM2016-79400-P and ICMAT Severo Ochoa project SEV-2015-0554 and the DFG project SFB 1085 'Higher Invariants'.

\section{References}

[1] O. Amini, S. Bloch, J. Burgos Gil and J. Fresán, 'Feynman amplitudes and limits of heights', Izv. Math. 80(5) (2016), 813-848.

[2] M. Asakura, 'Motives and algebraic de Rham cohomology', in The Arithmetic and Geometry of Algebraic Cycles (Banff, AB, 1998), CRM Proc. Lecture Notes, 24 (American Mathematical Society, Providence, RI, 2000), 133-154.

[3] O. Biesel, D. Holmes and R. de Jong, 'Néron models and the height jump divisor', Trans. Amer. Math. Soc. 369 (2017), 8685-8723.

[4] C. Birkenhake and H. Lange, Complex Abelian Varieties, 2nd edn, Grundlehren der Mathematischen Wissenschaften, 302 (Springer, Berlin, 2004).

[5] S. Boyd and L. Vandenberghe, Convex Optimization, (Cambridge University Press, Cambridge, UK, 2009).

[6] P. Brosnan and G. Pearlstein, 'Jumps in the Archimedean height', Preprint, 2017, arXiv:170 1.05527.

[7] J. I. Burgos Gil, D. Holmes and R. de Jong, 'Positivity of the height jump divisor', Int. Math. Res. Not. IMRN; doi:10.1093/imrn/rnx169. 
[8] J. I. Burgos Gil, J. Kramer and U. Kühn, 'The singularities of the invariant metric on the line bundle of Jacobi forms', in Recent Advances in Hodge Theory, (eds. M. Kerr and G. Pearlstein) London Mathematical Society Lecture Note Series, 427 (Cambridge University Press, Cambridge, 2016), 45-77.

[9] E. Cattani, 'Mixed Hodge structures, compactifications and monodromy weight filtrations', in Topics in Transcendental Algebraic Geometry, (ed. P. Griffiths) Ann. of Math. Stud., 106 (Princeton University Press, Princeton, NJ, 1984), 75-100.

[10] P. Deligne, 'Le déterminant de la cohomologie', in Current Trends in Arithmetical Algebraic Geometry (Arcata, CA, 1985), Contemporary Mathematics, 67 (American Mathematical Society, Providence, RI, 1987), 93-177.

[11] P. Deligne, Equations différentielles à points singuliers réguliers, Lecture Notes in Mathematics, 163 (Springer, Berlin, 1970).

[12] A. Grothendieck, Groupes de monodromie en géométrie algébrique, Lecture Notes in Mathematics, 288 (Springer, Berlin, 1972).

[13] R. Hain, 'Biextensions and heights associated to curves of odd genus', Duke Math. J. 61 (1990), 859-898.

[14] R. Hain, 'Normal functions and the geometry of moduli spaces of curves', in Handbook of Moduli, Volume I, (eds. G. Farkas and I. Morrison) Advanced Lectures in Mathematics, XXIV (International Press, Boston, 2013).

[15] T. Hayama and G. Pearlstein, 'Asymptotics of degenerations of mixed Hodge structures', Adv. Math. 273 (2015), 380-420.

[16] D. Holmes and R. de Jong, 'Asymptotics of the Néron height pairing', Math. Res. Lett. 22(5) (2015), 1337-1371.

[17] M. Kashiwara, 'A study of variation of mixed Hodge structure', Publ. RIMS, Kyoto Univ. 22 (1986), 991-1024.

[18] D. Lear, 'Extensions of normal functions and asymptotics of the height pairing', $\mathrm{PhD}$ Thesis, University of Washington, 1990.

[19] G. Pearlstein, 'Variations of mixed Hodge structure, Higgs fields, and quantum cohomology', Manuscripta Math. 102(3) (2000), 269-310.

[20] G. Pearlstein, 'SL $\mathrm{SL}_{2}$-orbits and degenerations of mixed Hodge structure', J. Differential Geom. 74 (2006), 1-67.

[21] G. Pearlstein and C. Peters, 'Differential geometry of the mixed Hodge metric', Commun. Anal. Geom. (to appear), Preprint, 2014, arXiv:1407.4082.

[22] C. Peters and J. Steenbrink, 'Mixed Hodge structures', in Ergebnisse der Mathematik und ihrer Grenzgebiete. 3. Folge, A Series of Modern Surveys in Mathematics, 52 (Springer, Berlin, 2008).

[23] R. T. Rockafellar, Convex Analysis, Princeton Mathematical Series, 28 (Princeton University Press, Princeton, 1970).

[24] M. Saito, 'Modules de Hodge polarisables', Publ. Res. Inst. Math. Sci. 24 (1988), 849-995.

[25] M. Saito, 'Mixed Hodge modules', Publ. Res. Inst. Math. Sci. 26 (1990), 221-333.

[26] J. Steenbrink and S. Zucker, 'Variation of mixed Hodge structure. I', Invent. Math. 80 (1985), 489-542. 